\section{Mobile Devices}

More than just a cellular phone?

The Application Developer's Perspective

Einar Lück,

Konrad Hübner,

HASSO - PLATTNER- H

Robert Mitschke INSTITUT

\section{Agenda}

- Introduction, Motivation, Overview

- Problems implied by Mobile Devices

- Programming Challenges

- Infrastructure, Platforms, Programming Models - Available Platforms

- J2ME

Blackberry Programming Practice

- Blackberry Platform Characterization \& API Introduction

- Communication \& Synchronization

- Portability

- Additional Features

* The software engineering process in the mobile world

- Requirements Analysis

- Design (with focus on mobile world constraints)

- Implementation (IDEs for development for mobile systems)

- Testing (additional test demands, tools for simulation)

- Deployment

nents Programming \& Middleware, Mobile Devices/ 2 HASSO - PLATTNER-INSTITUT P

\section{The Mobile World}

\section{- Perspective}

- Application Development for Mobile Devices

- Motivation for Applications on Mobiles

- Ubiquitous computing

- Stay connected (with business or friends)

- Diversify communication methods

- Retrieve Information

- Personal Information Management (PIM)

- Fight boredom

- Impress girls

+ Use cases

- Mail, News, Calendar, Contacts

- Business Applications (access intranet applications ...)

- Check, Buy and Sell stock (time critical application)

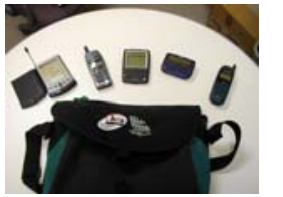

- Play games, Download \& Play music

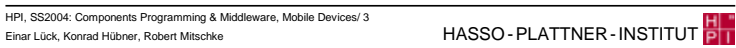

\section{The Mobile World - Development Environment}

\section{* Major differences}

- Development Device != Target Device

$\Rightarrow$ Development Architecture != Target Architecture

- Demand for Cross-Compiler

- Demand for deployment facility (e.g. IrDA, Bluetooth, Provision over air)

- Simulators may be useful

- Remote debugging facility useful
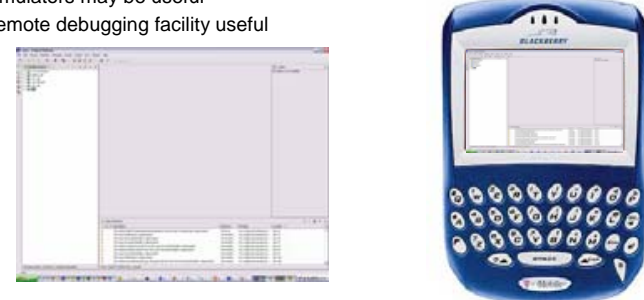

HPI, SS2004: Components Programming \& Middleware, Mobile Devices/4

Einar Lück, Konrad Hubner, Robert Mitschke

\section{Problems implied by Mobile Devices}

* Resource-poor compared to Desktops, Servers

\begin{tabular}{|c|c|c|c|}
\hline & $\begin{array}{c}\text { Genuine Cell } \\
\text { Phone }\end{array}$ & Smartphone or PDA & Average Desktop \\
\hline $\begin{array}{c}\text { Processing } \\
\text { Power }\end{array}$ & $12-30 \mathrm{MHz}$ & $50-200 \mathrm{MHz}$ & $2, \times \mathrm{GHZ}$ \\
\hline $\begin{array}{c}\text { Volatile } \\
\text { Memory (RAM) }\end{array}$ & $512 \mathrm{~Kb}-1 \mathrm{MB}$ & $2-16 \mathrm{MB}$ & $256 \mathrm{MB}-1 \mathrm{~GB}$ \\
\hline $\begin{array}{c}\text { Non volatile } \\
\text { Memory }\end{array}$ & $\begin{array}{c}2-4 \mathrm{MB} \\
\text { (mostly Flash) }\end{array}$ & $\begin{array}{c}16-512 \mathrm{MB} \\
\text { (depending on add- } \\
\text { on memory card) }\end{array}$ & $60-100 \mathrm{~GB}$ \\
\hline
\end{tabular}

- Usually limited access to additional hardware or interfaces (e.g. Bluetooth, IRDA, RS232)
Problems implied by Mobile Devices (cont.)

- Connectivity variability in performance and reliability

- Lack of reliability due limited coverage areas

- Bandwidth variations due to different (wireless) connections

\begin{tabular}{|l|c|c|c|c|}
\hline & GSM & GPRS & UMTS & WLAN \\
\hline Switched & $\begin{array}{c}\text { Circuit } \\
\text { Switched }\end{array}$ & $\begin{array}{c}\text { Packet } \\
\text { Switched }\end{array}$ & $\begin{array}{c}\text { Packet } \\
\text { Switched }\end{array}$ & $\begin{array}{c}\text { Packet } \\
\text { Switched }\end{array}$ \\
\hline Bandwidth & $14,4 \mathrm{kBit}$ & $56 \mathrm{~kb} / \mathrm{s}$ & $2 \mathrm{MBit}$ & $54 \mathrm{Mbit}$ \\
\hline
\end{tabular}

+ Finite energy sources

- Power consumption / duration of battery lifetime is key marketing criterion

- Applications should not consume much power

- Applications may help in managing power

- Applications should be aware of power consumption of hardware in use

(Bluetooth, IRDA, RS232, Screen lighting, Network) 
Problems implied by Mobile Devices (cont.)

+ Demand for synchronization

- Limited connectivity, bandwidth and power

- Mobile not be accepted as storage platform for information

$\rightarrow$ Information needs to be stored locally which requires synchronization

- Synchronization demand crosses application domains

(e.g. Mail \& News, Contacts, Web Pages, Calendar, Business Data, Files)

- Security threats

- Malicious Code

- May secretly use the device and incur costs

May make the device unusable

- Networking related threats

- Eavesdropping attacks

- Replay attacks

$\rightarrow$ used to gain secret information

HPI, SS2004: Components Programming \& Middleware, Mobile Devices/ HASSO-PLATTNER-INSTITUT
Problems implied by Mobile Devices (cont.)

- Variety of hardware and APIs (Portability and Ergonomics)

- Different screen dimensions

- Different screen colour depth

- Different input means (ranging from knobs and wheels to pointing devices)

- GUI constraints (e.g. senseless: variable window sizes)

- Different APIs (e.g. native, Java, .NET)

$\rightarrow$ Contradicts application portability across different devices

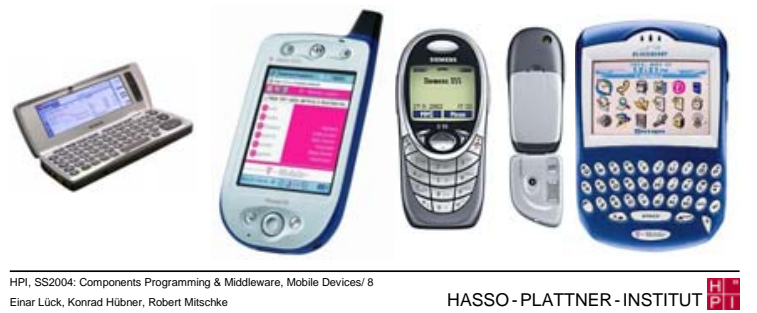

\section{Application Programming Challenges}

\section{+ The Key to Mobility: Adaptation [6]}

- Lack of local resources: server based application logic / data storage

- Lack of reliability: mobile based application logic / data storage

\section{Problems}

- Adaptation may be difficult to implement

- Similarities in adaptation between different applications

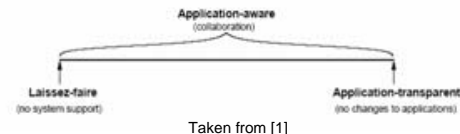

Einar Luck, Konrad Hubner, Robert Mitschke

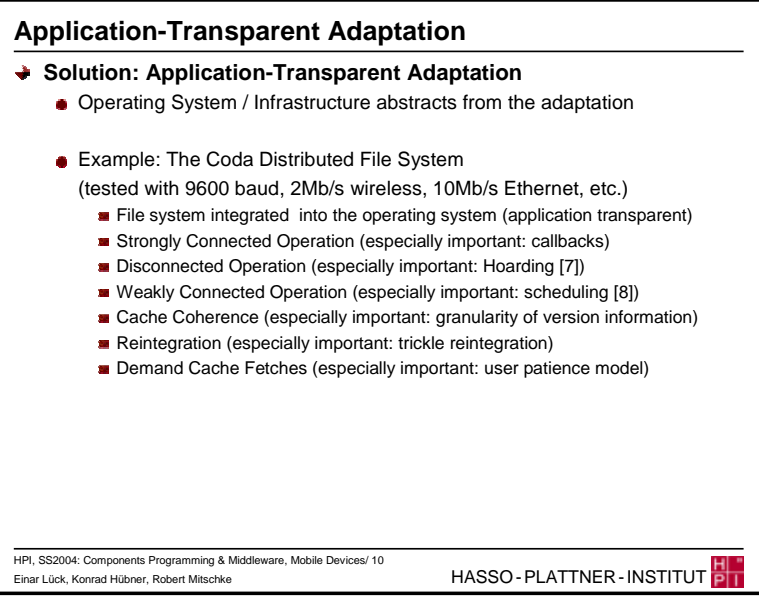

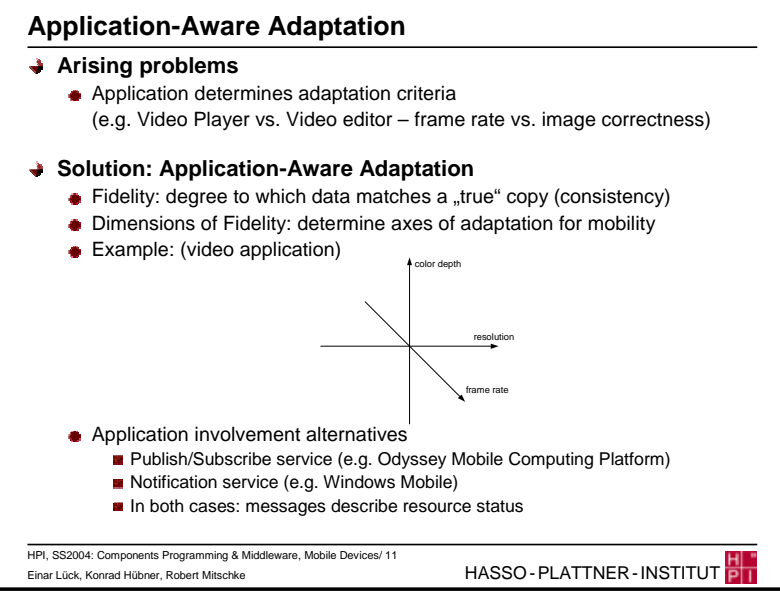

\begin{tabular}{l} 
Programming Challenges - Categorization \\
- Sommunication \\
Network imposed problems \\
Device Limitations \\
- Power consumption \\
- Memory restrictions \\
Storage capacity \\
Security Requirements \\
- Malicious code \\
- Eavesdropping attacks \\
- Platform diversity \\
- Screen and input device diversity \\
\hline HPI, SS2004: Components Programming \& Middleware, Mobile Devices/12 \\
Einar Luck, Konrad Hubner, Rober Mischke
\end{tabular}



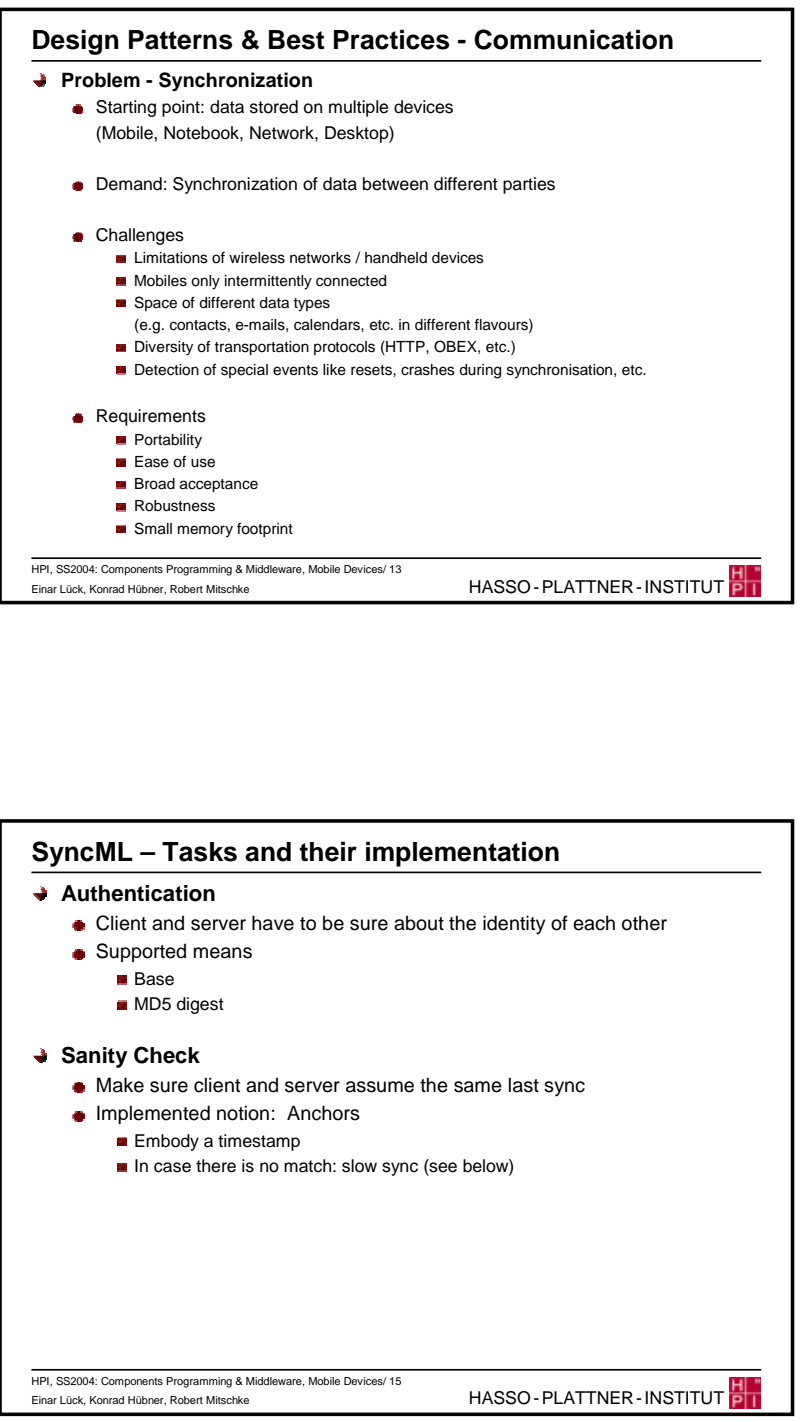

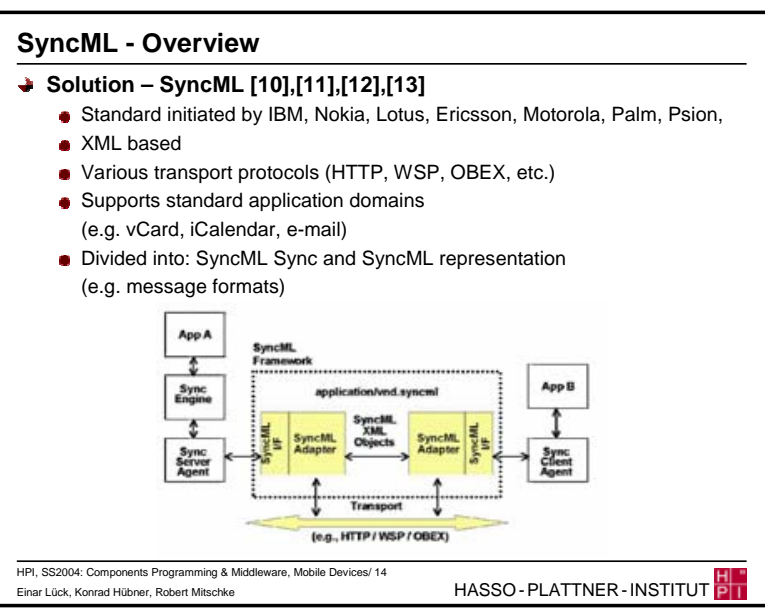

SyncML - Tasks and their implementation

- Exchange of Capabilities, Methods and Database

- Not all methods are support by all clients and servers

- Required by the protocol in the exchange phase

\section{- Data Item Mapping}

- Map data item on clients to corresponding data items on server

- Notion of Unique Identifiers

- Local Unique Identifier (LUID) identifies items on client

- Global Unique Identifer (GUID) identifies items on server

- Important: Mapping maintained by server

- LUIDs are always assigned by by the client, GUIDs by the server

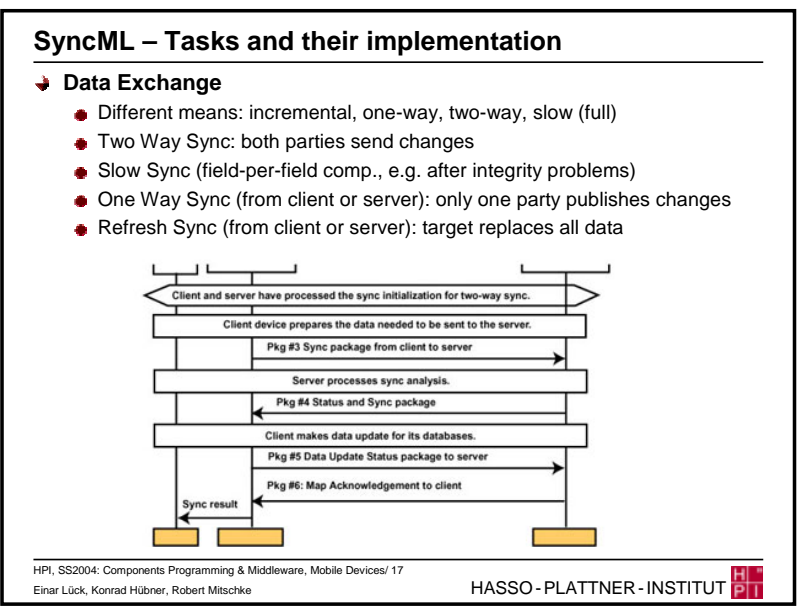

SyncML - Tasks and their implementation

\section{* Conflict Resolution}

- Restricted to conflict detection

- Resolution kept out of protocol

- Application domain support

- Support for everything serialized in XML

- Vcard, iCalendar, etc.

Keep track of changes and possible problems

- Maintain a change log

- Precise format not specified 


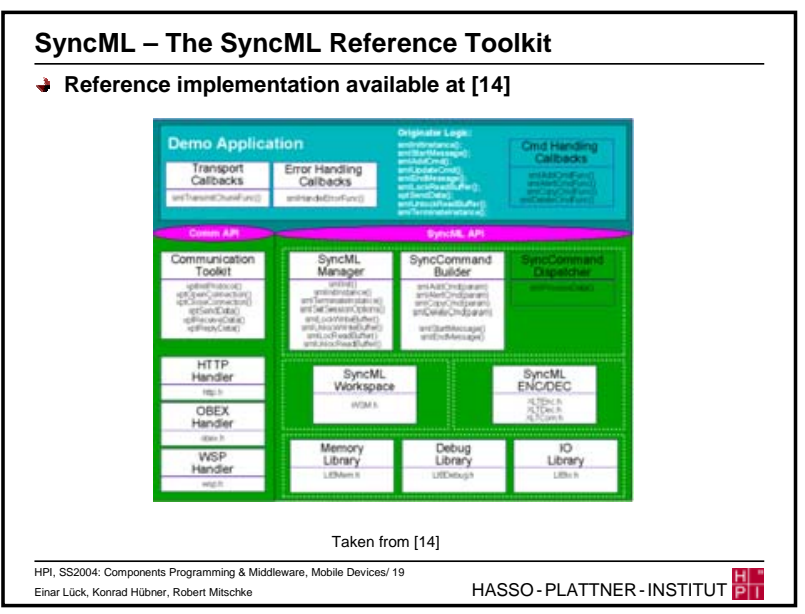

SyncML - A Small example

+ TODO
SyncML - Reference Toolkit - Example API

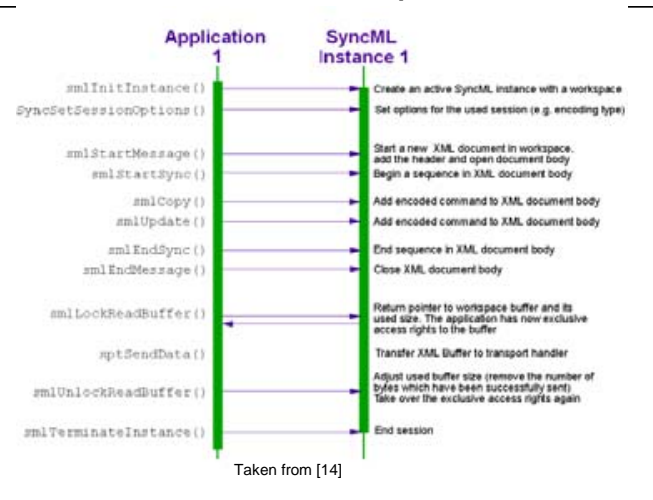

HPI, SS2004: Components Programming \& Middleware, Mobile Devices/ 20
Einar Lick, Konrad Hüner, Robert Mitschke

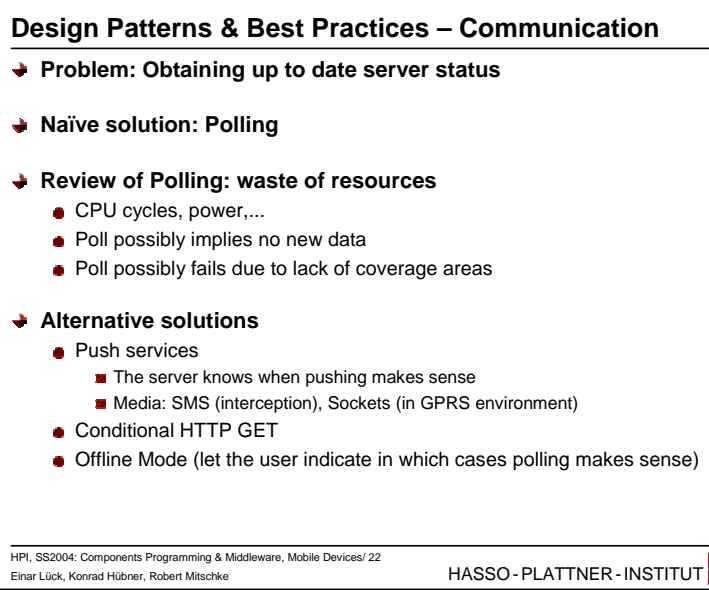

Design Patterns \& Best Practices - Communication

+ Problem: Obtaining up to date server status

+ Naïve solution: Polling

- Review of Polling: waste of resources

- CPU cycles, power,...

- Poll possibly implies no new data

- Poll possibly fails due to lack of coverage areas

- Alternative solutions

- Push services

- The server knows when pushing makes sense

$\square$ Media: SMS (interception), Sockets (in GPRS environment)

- Conditional HTTP GET

- Offline Mode (let the user indicate in which cases polling makes sense)

HPI, SS2004: Components Programming \& Middleware, Mobile Devices/ 22

Einar Luck, Konrad Hüber, Rober Mitschke

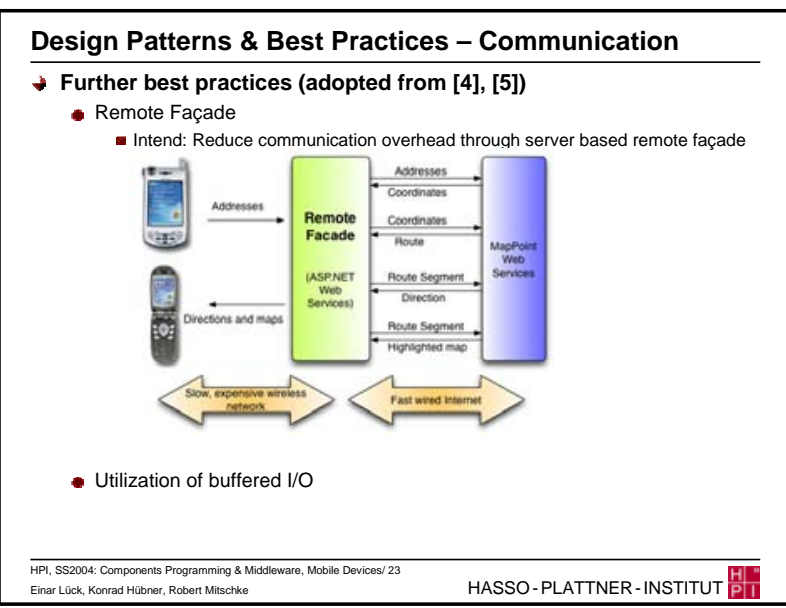

Design Patterns \& Best Practices - Memory Consumption

\section{- Application Example}

- Location Based Service visualizing options available in a certain area

- Naïve object/memory layout

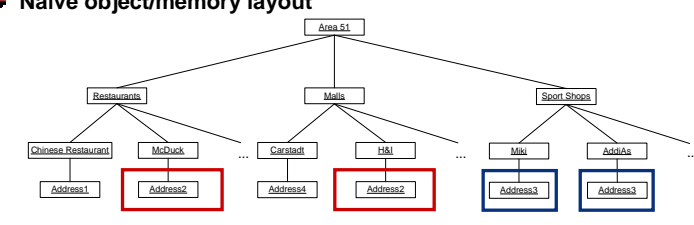

- Problems

- High correlation of address data not fostered

- Waste of memory

HPI, SS2004: Components Programming \& Middleware, Mobile Devicess 24 HनI, SS2004: Componentsts Programming \& M
Einar Lück, Konrad Hüber, Robert Mitschke 


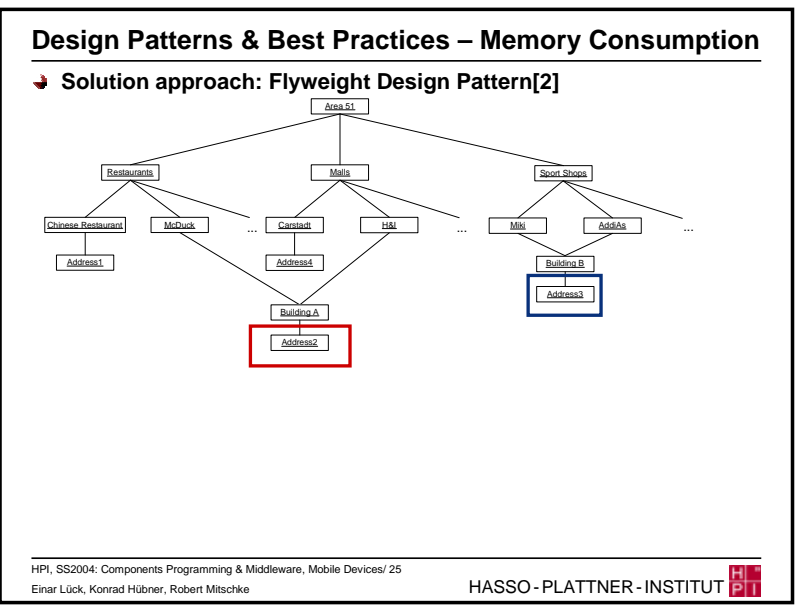

Design Patterns \& Best Practices - Memory Consumption

- Examples for further best practices (according to [4])

- Keep Garbage Collection at a minimum

- Arrays instead of collection classes

- Free resources as fast as possible (sockets, files, etc.)

- Reduce Application Footprint

- Partition the system

- Optimize Packaging (only package stuff always utilized concurrently)

- Avoid excessive layering

- Utilize application domain knowledge (e.g. Computer Graphics [3])

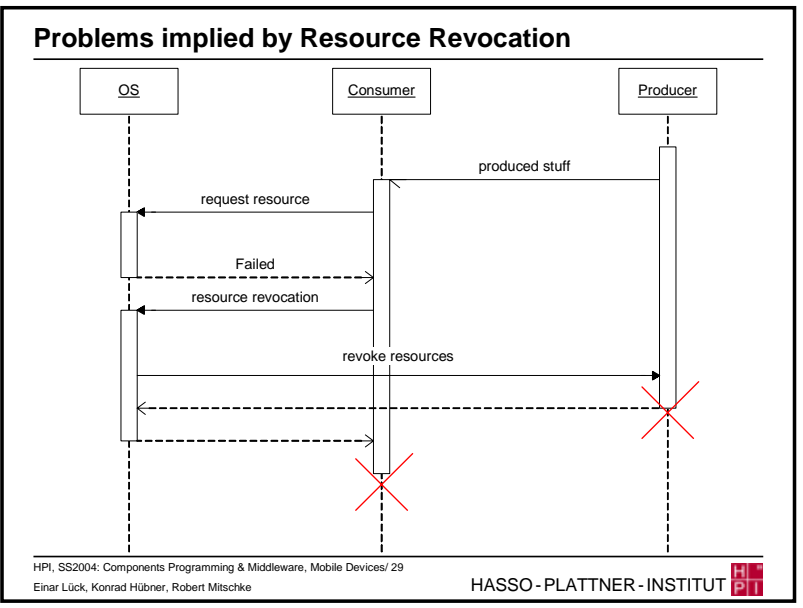

Design Patterns \& Best Practices - Memory Consumption

- Applicability (adopted from [2])

- Application uses large number of objects

- Storage costs are high

- Many groups of objects may be replaced by relatively few shared objects

- Review of Flyweight Design Pattern

- Requires application developers reconsideration

- Rather straightforward in application

- Domain independent

Design Patterns \& Best Practices - Memory Consumption

- Memory saving approach on OS level - Resource Revocation

(e.g. memory, file descriptors, sockets, DB connects)

- OS may revoke resources from other applications (on request)

- Possibly arising problem: Deadlocks, Stalls

- Workarounds

- Allow victim applications to decide on revocation

- Let applications mark objects that may be removed in case of bottle necks

Design Patterns \& Best Practices - Security

+ Problem

- Malicious code may cause costs or may destroy important data

- Solution: Application Signing

- Code has to be signed to access certain APIs

- Basis: Asymmetric cryptography algorithm

- Signing: Encryption with a private key only known by a defined party

- Further Problem

- Eavesdropping attacks

- Of major importance as in most cases business or PI are affected

- Solution: Cryptology

- Utilization of established cryptology technologies

(e.g. HTTPS, Triple DES, SSL/TLS) 


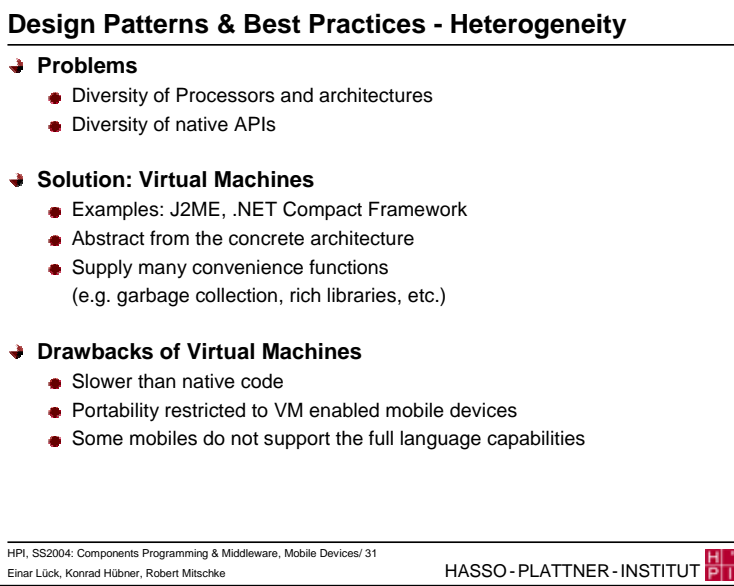

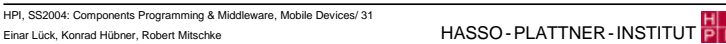

Design Patterns \& Best Practices - Heterogeneity

- Alternative Solution: Application Frameworks and cross compilers

- Examples: Qtopia from Trolltech (Linux based) [9]

- Class libraries abstracting from platform specifics

- Drawbacks of Application Frameworks and cross compilers

- Not suitable for application domains

- Restricted availability across different platforms / Implementation Overhead

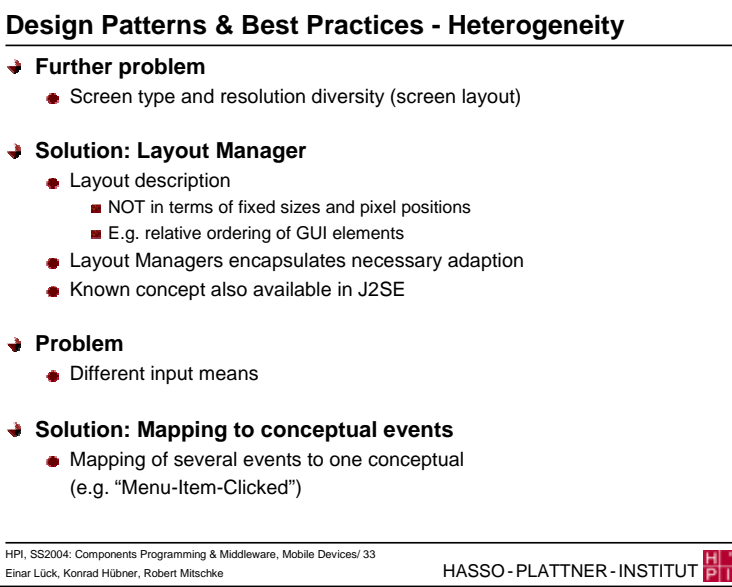

- Screen type and resolution diversity (screen layout)

\section{- Solution: Layout Manager}

- Layout description

NOT in terms of fixed sizes and pixel positions

- E.g. relative ordering of GUI elements

- Layout Managers encapsulates necessary adaption

- Known concept also available in J2SE

+ Problem

Different input means

+ Solution: Mapping to conceptual events

- Mapping of several events to one conceptual

(e.g. "Menu-Item-Clicked")

Summary

- Arising demand for mobile applications

+ Problems implied by Mobile Devices

- Device resource limitations (Power, CPU, Memory, etc.)

- Diversity of Input/Output means

Unreliability network

\section{- Programming Challenges}

- Key aspect: Adaptation

- Application-Aware Adaptation (e.g. video application)

- Application-Transparent Adaptation (e.g. distributed file system)

- Design Patterns and best practices

- Very helpful to have in mind when starting to develop

- Communication:

Synchronisation (SyncML), Push, Conditional Get, Remote Facade

- Memory consumption: Flyweight Pattern, Resource Revocation

- Security: Application Signing, Cryptology

- Heterogeneity: Virtual Machines, Application Frameworks, LayoutManager

HPI, SS2004: Components Progranming \& Middleware, Mobile Devices/ $34 \quad$ HASSO - PLATTNER-INSTITUT H|

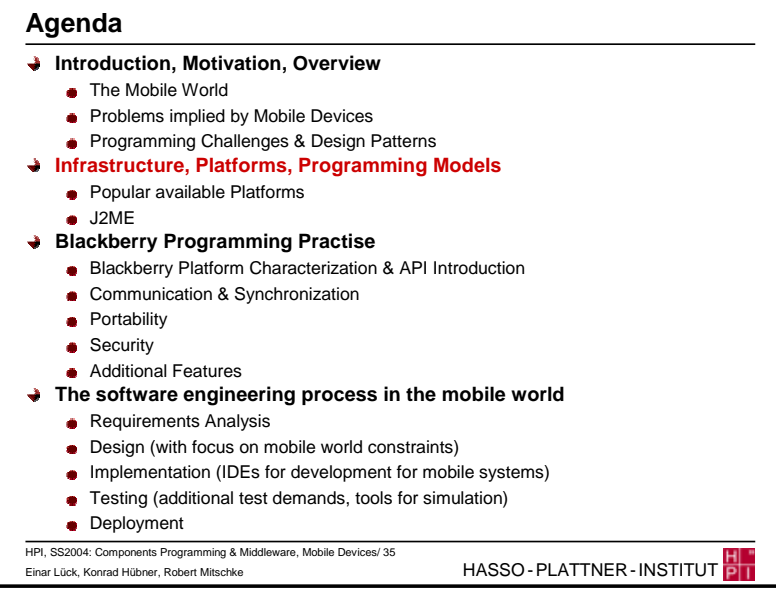

Popular available Platforms

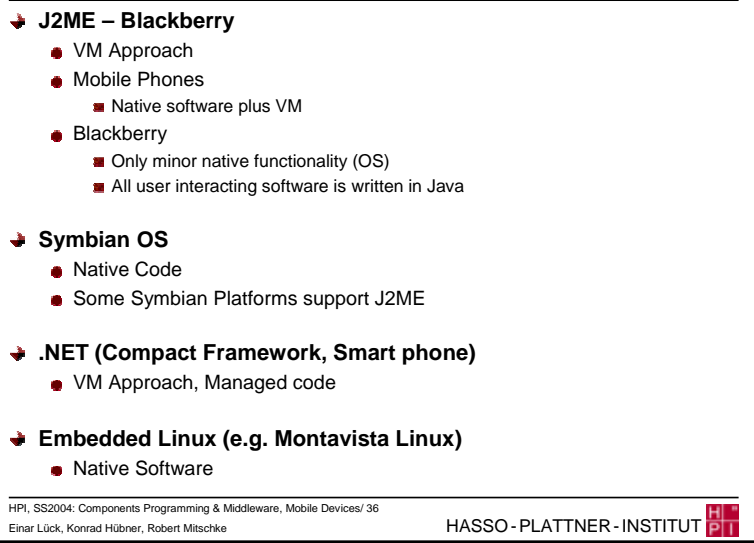




\section{J2ME - Java 2 Micro Edition}

Java for small devices

- Simplifies Portability

- Standardization at high level allows broad range of hardware platforms

- Streamlines Application development

J2ME is a framework

- Designed to support different device classes like

Parking meter

Mobile phone

Watch

Impose different requirements

- Thus a J2ME runtime environment consists of multiple building blocks HPl, SS2004: Components Programming \& Middleware, Mobile Devices/ 37
Einar Lük, Konrad Hubner, Robert Mischkee
J2ME - Architecture

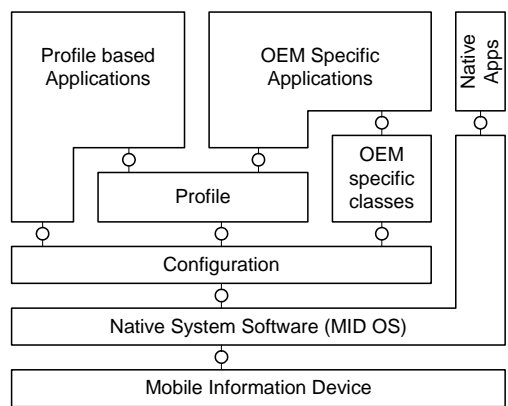

J2ME environment:

- Profile + Configuration + Virtual Machine = Java Technology Stack

API, SS2004: Components Programming \& Middleware, Mobile Devices/ 38
Einar Luck, Konrad Hüber, Robert Mitschke

\section{J2ME - Configuration}

\section{Configuration}

- "A J2ME configuration shall only define a minimum complement or the "lowest configuration must be ge Java technology. All he features included in a a profile rather than in a configuration. This means that the scope of a configuration is limited and must generally be complemented by profiles."

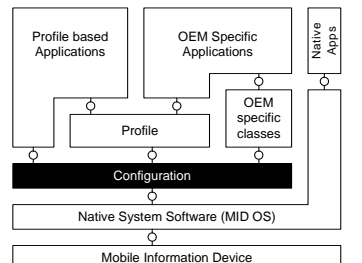

\section{Mobile Information Device}

J2ME - Configuration cont.

\section{+ Defines:}

- Application Management (Retrieving, Loading \& Executing)

- Low Level Security Measures (e.g. Byte Code Verification)

- Available Byte Code Instructions (Usually Subset of J2SE)

- Class File Format

- Mandatory available Libraries

- This is just a basic set, additional libraries are specified in Profile

- May not define optional features!!

- Example Configuration:

CDLC (Connected Limited Device Configuration)

HPI, SS2004: Components Programming \& Middleware, Mabile Devices/ 40

Einar Luck Konrad Hübner. Robert Mitschle HASSO-PLATTNER-INSTITUT

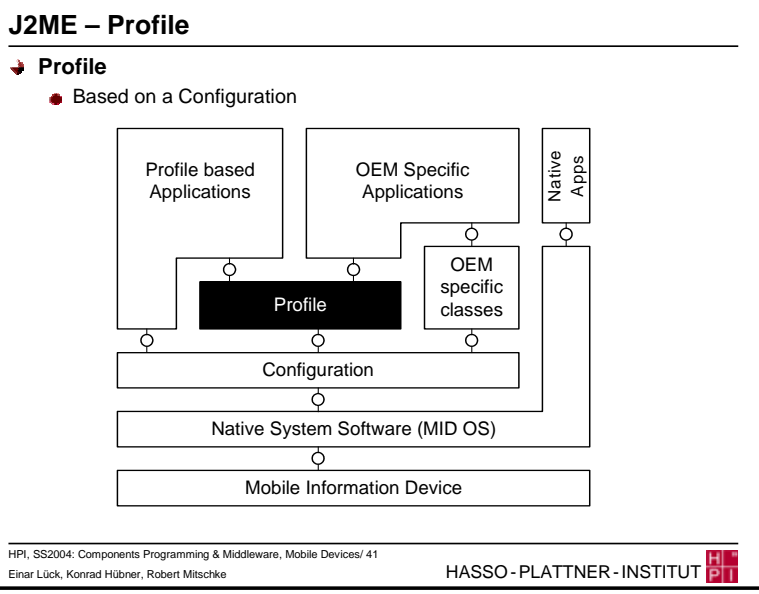

\section{J2ME - Profile cont.}

* Completes runtime environment specification

- Specifies additional hardware constraints

- Specifies API available additional to that specified by Configuration

- Intensely narrows down specification of target device

- Still even though target platform is narrow, a Profile is targeted at a whole class of devices, not at a specific platform

- E.g. MIDP is used on all mobile phones supporting Java, even on some Smart Phones and/or PDAs (Mobile Information Devices)

\section{- Example Profile:}

MIDP (Mobile Information Device Profile) 


\section{J2ME - CLDC a sample Configuration}

\section{+ CLDC - Connected Limited Device Configuration}

- Currently two versions (1.0 \& 1.1)

- 1.1 is backward compatible (1.0 Applications will run on 1.1 System)

- Hardware requirements

128KB non volatile memory for Virtual Machine and libraries

- 32KB RAM for java runtime and object memory

$\rightarrow$ Almost no sensible application will run with that amount of memory though

HP1, SS2004: Components Programming \& Middleware, Mobile Devices/

Einar Lück, Konrad Hüner, Robert Mitschke HASSO-PLATTNER-INSTITUT PH
J2ME - CLDC - OS Requirements

+ os requirements

- One schedulable entity

- Thus underlying system does not need to provide multi-threading/processing

- No requirements for

aneal time

- Separate address spaces

- Latency behaviour

$\rightarrow$ security is sacrificed for wider target device range

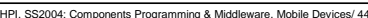

Einar Lickck, Konrad Hübrer, Bobert Mitschle HASSO-PLATTNER-INSTITUT P

\section{J2ME - CLDC - Application Management}

\section{* Application Management}

- No persistent storage required for applications - However it is strongly suggested

- Without storage apps could be loaded on demand

- Management of storage and application start up is considered out of scope - Underlying platform has to supply means for the user to execute apps - No assumptions about possible file systems etc.

\section{J2ME - CDLC - Connection Framework}

* Connection Framework of CLDC

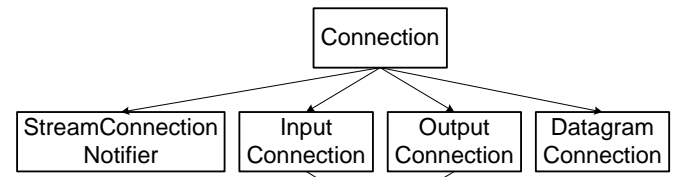
Notifier

\section{J2ME - CLDC compared to J2SE}

\section{- Subset of J2SE}

- No floating point support

Byte code instructions removed

was reintroduced in CDLC 1.1

- No Java Native Interface (JNI, calling of native c-functions)

- No user defined class loaders (security)

- No reflection

- No Thread groups

- Multithreading is supported, thread groups may be implemented by application developer

- No finalization

- No weak references

- references not counting as references for Garbage collector

- Reintroduced in CLDC 11

- Limited error handling - exceptions do exist though

J2ME - CDLC - Connection Framework

- Key ingredient of CLDC

- Connector.open ("<protocol>:<address $>$; $<$ parameters $>$ ") ;

- May potentially be used for

- HTTP

- Bluetooth, RS232

- TCP, UDP

Any other unidirectional

or bidirectional stream-

or datagram-based

No implementation of any protocol contained in CLDC $\rightarrow$ Task of Profile

- Allows broad functionality

- Any known protocol or communication technique may be supported

- Saves non volatile memory (flash)

- Abstract interface in Java potentially covers any protocol

- Lightweight implementation in platforms own native code

ipl SS2004: Components Prosemming \& Middleware, Mobile Devices/ 48

HASSO-PLATTNER-INSTITUT H 


\section{J2ME - CDLC - Security}

\section{Security}

- Byte code Verification divided into two step - process

- Out of device verification at compile time

- When compiling code is pre verified

- Slightly enlarges code size

- binary code is restructured and attributes are added

- Pre verification is semantically verified by runtime verifie

- Exploit using fake pre verification should not work

- Shortened verification at runtime

- Due to attributes in binary code only a single run is required

- This is not memory intensive due to pre verification

- Simple linear scan

- 10kbyte of x86 Code and 100bytes of RAM required on device

$\rightarrow$ Roughly: No illegal byte code or code violating type safety can be executed HPl, SS2004: Components Programming \& Middleware, Mobile Devices/49
Einar Luck, Konrad Hubner, Robert Misschke
J2ME - MIDP a sample Profile

- MIDP - Mobile Information Device Profile

- Two Versions available

- MIDP 1.0

- MIDP 2.0

- Based on CLDC

- MIDP 1.0 is supposedly based on CLDC 1.0

- MIDP 2.0 is supposedly based on CLDC 1.1

- Created for cellular phones, two way pagers, wireless enabled PDAs

- Created by MIDPEG (MIDP Expert Group)

- Instead of Applet or Servlet, Applications are called MIDlet

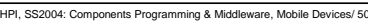

Einar Lück, Konrad Hübner, Robert Mitschke HASSO-PLATTNER-INSTITUT

\section{J2ME - MIDP Requirements}

- Requirements on top of CDLC

- Hardware

- 96×54 pixel screen dimension, 1 Bit colour depth

ITU-T Keyboard (Phone keyboard), QWERTY Keyboard and/or touch screen

- 128kb additional non volatile memory for MIDP components

- 8kb non volatile memory for application created persistent data

- $32 \mathrm{~kb}$ volatile memory for Java runtime

- Two way, wireless, potentially unstable, low bandwidth connectivity

- For comparison

.Net Compact Framework requires multiple Megabytes of volatile and non volatile memory

\section{J2ME - MIDP Requirements}

\section{+ Operating System must support}

- Writing to persistent storage (for app created data, not necessarily apps)

- Access to networking hardware

- Time base

- Access to bit mapped display

- Access to user input

\section{J2ME - MIDP Connection Framework support}

\section{Connection Framework}

$\rightarrow$ CLDC did not require any protocols, just supplied the Framework

+ MIDP requires HTTP

- May base on Gateway

- Device does not need to implement full IP Stack

- May use arbitrary protocol, in case Gateway supplies IP Stack,

like in WAP or switched circuit

\section{J2ME - MIDP Connection Framework deficiencies}

- MIDP does not require other protocols

- No socket

- No datagram based communication

- No required communication via other interfaces (Bluetooth, IRDA, ...)

- Portable applications have to rely on HTTP only

- SyncML is not part of MIDP $\rightarrow$ Has to be implemented individually 


\section{J2ME - MIDP - Persistent Storage}

\section{+ Persistent Storage}

- Allows storage of application data in so called record stores

- Does not require file system, abstract data store

- Automatic serialization of write requests

- Application does not have to take care of organizing concurrent write requests

private RecordStore recordStore $=$

RecordStore.openRecordstore("nirwana", true);

byte[] b = myChunkofInfo.toByteArray ( )

recordStore.addRecord (b, $\odot$, b.length);

- Additionally supports enumeration, filtering

- Looks like it was made to access high score tables (-)
J2ME - MIDP - Display Capabilities

\section{+ Display Capabilities}

- Probably most important part of the API

- Applications are probably GUI-heavy as platform is not useful for heavy computing

- Most applications will require intensive user interaction

- API is split into two parts

- Highly Abstract API (called High level API)

- Abstracts from all direct screen and input device access

- Low Level API (called Low Level API (-))

- Allows access to the screen and input devices

\section{J2ME - MIDP - High Level GUI API}

\section{+ High level API}

- Abstracts from Screen and Input device capabilities

- Allows to display choices, forms

- The way these are displayed on the screen are implementation dependen - Device's own colour scheme is used

Information is displayed according to devices GUI scheme and capabilities

- Allows to listen to abstract commands

- Device decides on how to allow user interaction

Device should adhere to it's own scheme, which the user is accustomed to

- Application programmer does not have to worry about available display capabilities, available input devices

- Application just displays information and listens for returning events

- Widgets are : Lists, Selections, Gauge, Textbox ..

\section{J2ME - MIDP - Code Example High Level API}

mMainForm = new Form("Available Memos")

mMainForm. addCommand (ok = new Command ("Weiter", Command.oK, 0$)$ ), mMainForm. addCommand (ok2 = new Command ("Nochweiter", Command.ox, 0$)$ );

(T)

mMainForm. setcommandListener(this)

d = Display.getDisplay(this) d. setcurrent (mMainForm)

public void commandAction (Command c, Displayable s) \{ if (c == exit) notifyDestroyed():

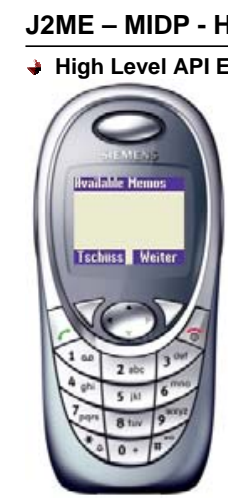

1 screen -2 commands HPl, SS2004: Components Programming \& Midd
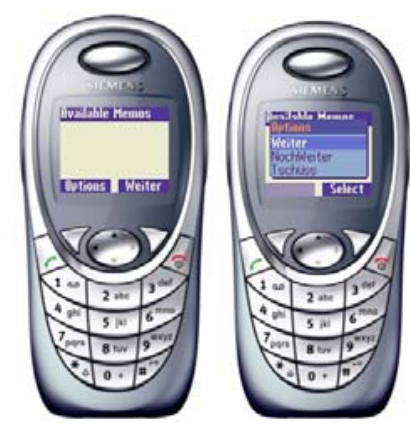

1 screen -3 commands
J2ME - MIDP - High Level API in action cont.

- High Level API Example - Sun's imaginary devices

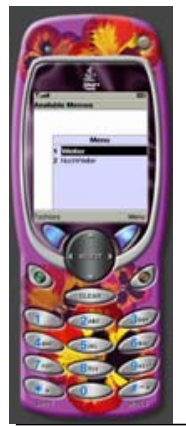
All: 1 screen -3 commands

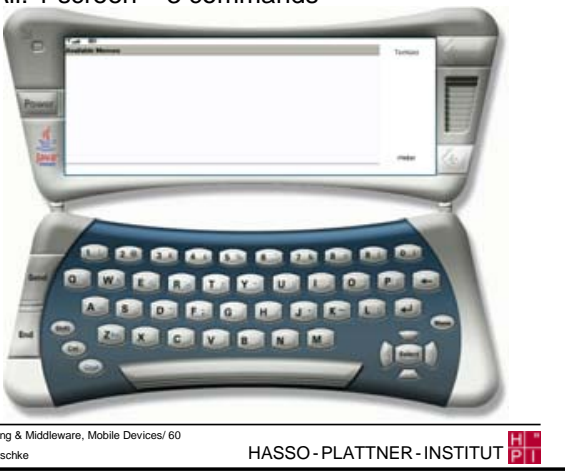




\section{J2ME - MIDP Low Level API}

Low Level API

- Allows direct access to display and input devices

- Pictures may be shown

- Pixels may be drawn using familiar commands (drawString ()$, \ldots$ )

- Application has to adjust to screen dimensions

- Application has to adjust to input devices

- Means to find present input devices exist

Application has to adjust its behaviour
J2ME - MIDP - Code Example Low Level API

class TextCanvas extends Canvas

\{

protected void paint (Graphics g) \{

int height $=$ getHeight (

int width = getwidth ()

if (hasPointerEvents ())

// support the input device pointer

if (hasPointerMotionEvents ())

// prepare for motion events

if (isDoubleBuffered ())

// take advantage of double buffered screen

// draw something that makes sense on the current platform

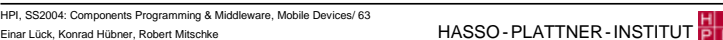

J2ME - MIDP Low Level API deficiencies

+ Mostly used for graphical game development

+ Applications using the Low Level API are either

- not portable at all

portable only with significant (non trivial)
Adjusting to screen size and colour depth - Adjusting to present input devices

- Performance may not be optimal due to portability

- Siemens provides own Game API to gain performance boost

(which is necessary on Siemens phones $\odot$ )
- Combination of Low and High-Level API is not intended

- But may work and impose security threat $\rightarrow$ see news on S55 SMS exploit

J2ME - MIDP - Application Management

+ Application Packaging \& Starting

- MIDlets are packet into so called MIDlet Suites

- One or more MIDlets including all their classes

- Resources used by the MIDlet(s)

- JAR-Manifest, containing information about all contained MIDlets and the

Suite itself

$\rightarrow$ MIDlet Suites are JAR-Files

- Selection and Starting of Applications

- Is out of the scope of the standard

- Is individually supplied by underlying operating system

\section{J2ME - MIDP - Application Management cont.}

- Application Descriptor (midletname.jad)

- File that additionally comes with the JAR

- Contains Information from the Manifest

- Optionally Contains Information on the location of the JAR (URL)

- Thus allows to retrieve Info before downloading the whole JAR

- Allows checking for updates and OTA Provisioning

MIDlet-1: Editor, Editor.png, Editor

MIDlet-Jar-Size: 2070

MIDlet-Jar-URL: Editor.jar

MIDlet-Name: Editor

MIDlet-Vendor: Unknown

MIDlet-Version: 1.0

MicroEdition-Configuration: CLDC-1.0

MicroEdition-Profile: MIDP-2.0

HPI, SS2004: Components Programming \& Middleware, Mobile Devices/

\section{J2ME - MIDP - Security concepts}

\section{- Security}

- Sandbox Concep

- Standard specifies all APIs available to MIDlet

- Additional functionality may not be accessible to MIDlet

- MIDlets may not access system functionality

- MIDlets may only communicate within MIDlet Suites and then only via Record

Store 
J2ME - MIDP - Security concepts cont.

- New in MIDP 2.0: Trust Model for sensitive (costly) functionality E.g. before a MIDlet may send HTTP Traffic (or SMS, ...) user would like to be asked

Organized in permissions and protection domains

- Using PKI MIDlets may be trusted without user interaction (optional)

HPI, SS2004: Components Programming \& Middleware, Mobile Devices HASSO-PLATTNER-INSTITUT P PII
J2ME - Summary

\section{+ Summarization}

- In our opinion J2ME is a step into the right direction

- CDLC + MIDP as used in most mobile phones today is not gone far enough

- Not enough functionality to implement the cool things

- Can mainly be used for games (Boredom can be overcome) (-)

- Portability is only ensured when sticking with the smallest common

denominator

- Girls may not be impressed :

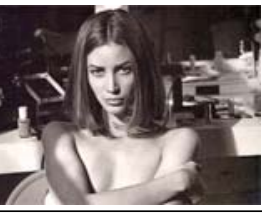

HPI, SS2004: Components Programming a

\section{J2ME - Outlook}

- CDLC + MIDP + OEM libraries can become powerful tools depending on the platform and the power of OEM libraries

- Basic functionality is common, short time to get familiar with a new platform

Now lets look at Blackberry, the Flagship of J2ME devices!!

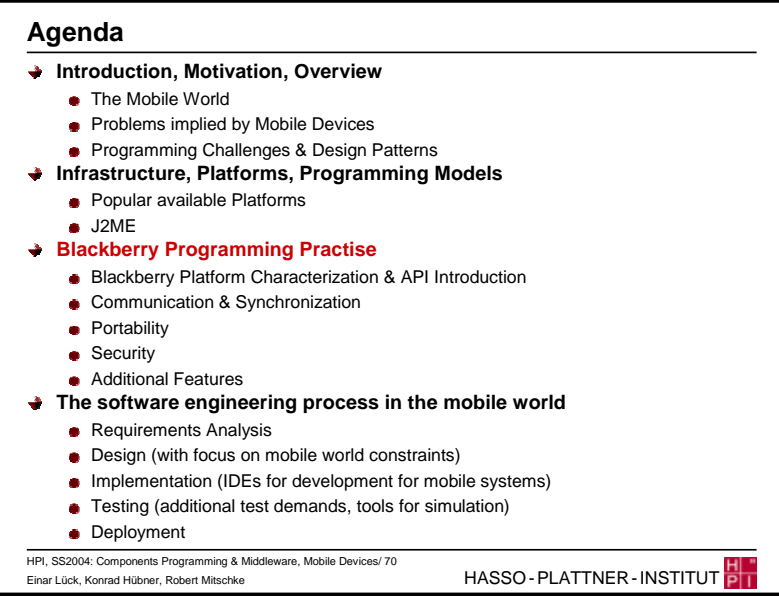

\footnotetext{
The Blackberry Platform - The Company

- Research in Motion

- founded 1984

- located in Canada (Waterloo, Ontario)

- Developer, manufacturer and marketer of wireless solutions

- RIM Inter@ctive Pager introduced in the mid-90s - Mobitex and DataTAC data networks in USA - Devices with TrackWheel and small keyboard

- In 1999 change of focus to

"Blackberry Wireless Email Solution" - Integration into corporate email system - Introduction of Java support

- Support for new networks like GPRS

- Newest models with phone support,

color display and 16MB flash memory
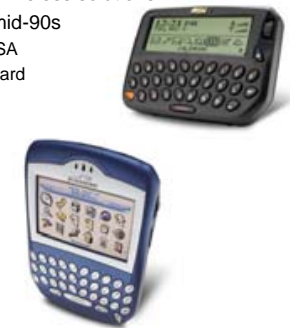

\section{The Blackberry Solution}

Middleware integrating Handhelds into corporate network

- Integrate into email system

- Support only for MS Exchange and Lotus Notes

- Gain access to company data 
The Blackberry Solution - Simple Architecture

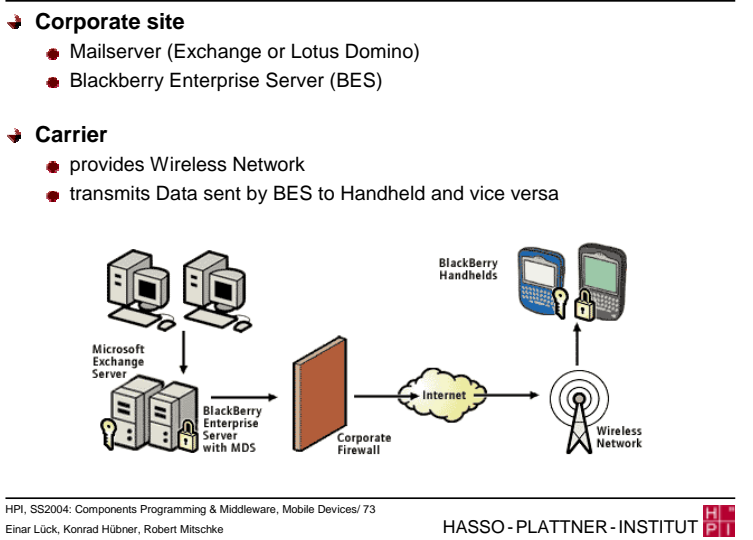

Einar Lück, Konrad Hüner, Robert Mistschke HASSO-PLATTNER-INSTITUT PII

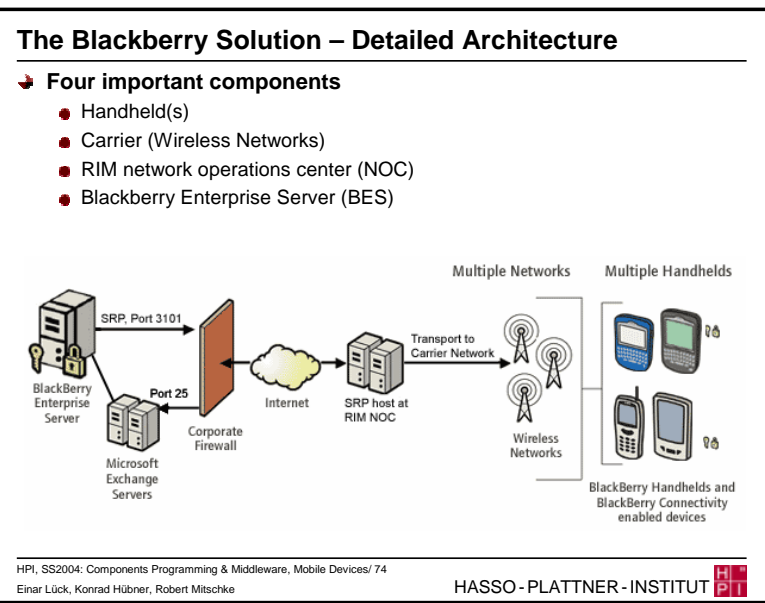

\section{The Blackberry Platform - BES}

\section{* Two-Way connection to NOC}

- Both Handheld and email-server have to access each other

- Handheld paired to exactly one BES

- NOC has to know all these linkings

- Encryption between BES and Handheld

Key generated by BES when Handheld is associated to BES

- Connected to NOC via permanent TCP Port 3101 connection - RIM's Service Relay Protocol (SRP) is utilized

- Mobile Data Service (MDS)

- Enables Handheld to receive data from the company network

- Provides internet access for Handheld

\section{The Blackberry Platform - NOC (cont.)}

* Handles all email traffic of the Handhelds

- Handhelds send email via carrier to NOC

- NOC forwards traffic to companie's mail server

- BES sends mails for Handheld to NOC which forwards it to the carrier

- Handles all data push traffic to Handhelds

- Forwards traffic received by MDS to Handheld

- Forwards traffic received by Handheld to MDS

The Blackberry Platform - NOC

\section{- Two NOCs worldwide}

- Waterloo, Ontario (Canada)

- Sterling, Virginia (US)

- Single point of connection for all Handhelds

- Handhelds registered with their email-address and id

- Unique association to a user

- Directly connected to all carriers supporting the Blackberry system

- Directly connected to all BESs installed worldwide

- TCP Port 3101 for incoming traffic by BES

- TCP Port 25 for traffic to company (SMTP Mailserver)

\section{The Blackberry Platform - Carrier}

Provides wireless network access to the Handheld

- Connected to RIM's NOC

- Provides additional internet access via WAP (in contrast to MDS)

- Provides phone services if supported by handheld 
The Blackberry Platform - Handheld Overview

+ email via BES Push-Service

- Handheld associated to exactly one BES and one user

- All emails delivered to and by this BES

+ email via other account (POP3)

- No BES needed

- No push-service possible

+ email via special account by carrier / RIM

- No BES needed

- Suiteable for personal users

- Push-service possible

- Device-User-Association required

HPl, SS2004: Components Programming \& Middleware, Mobile Devices/7 HASSO-PLATTNER-INSTITUT P
The Handheld - Details

- Old devices with C++ Interface

- Data services only

- Still available on market

- Application development more complex (i.e. Memory Management)

- Modern Blackberry Handhelds with Java

- Data and phone services

- Easy application development

- Garbage Collection leads to less memory consumption problems

\section{- Java VM}

- Developed by RIM

- Supports J2ME CLDC 1.0 and MIDP 1.0 specification

- Device-specific API for access to all Blackberry features

- Runs non-standard bytecode => RIM Development tools have to be used

ing \& Middleware, Mobile Devices/80

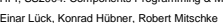
HASSO-PLATTNER-INSTITUT

\begin{tabular}{l} 
Communication Paradigms \\
Communication Means \\
HTTP(s) \\
Sockets \\
- Server-initiated connections to Handheld \\
Requires BES and MDS \\
Two available gateways \\
Blackberry Enterprise Server \\
- WAP gateway \\
Standard Communication routed through BES \\
- Seamless, no special connection estabishment to carrier needed \\
Connections via WAP gateway \\
- Specially formatted URLs necessary, containing WAP gateway information \\
\hline HP, SS2004: Components Programming \& Middleware, Mobile Devices/ 81 \\
Enar Luck, Konrad Hubner, Robert Mischke
\end{tabular}

\section{Application Portability}

\section{* J2ME CLDC I MIDP Applications}

- Portable to all JavaME capable devices

- Reach more possible customers

- Suffer from small functionality

\section{- Blackberry API Applications}

- Portable only to other Blackberry Devices

- Some features only on certain devices (Color vs BlackMhite)

- Provide more functionality to less customers

\section{Synchronisation Mechanisms}

Backup Handheld database through Desktop Software

- Database holding Application data is stored in a file on desktop system

- Data is transfered using bulk load

- Can be used to restore Application state on the handheld

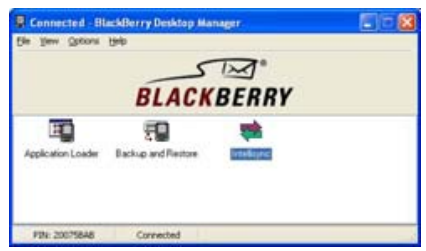




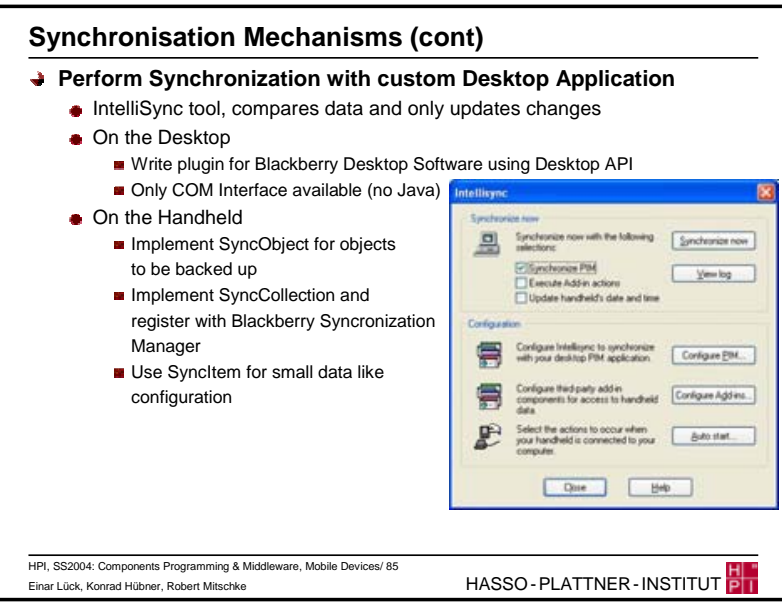

Synchronisation Mechanisms (cont)

ackberry Desktop Software using Desktop AP

com Interface available (no Java) intelling

- Implement SyncObject for objects

to be backed up

Use Syncltem for small data like

四

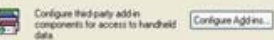

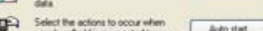

$\square \square$

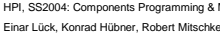
HASSO-PLATTNER-INSTITUT

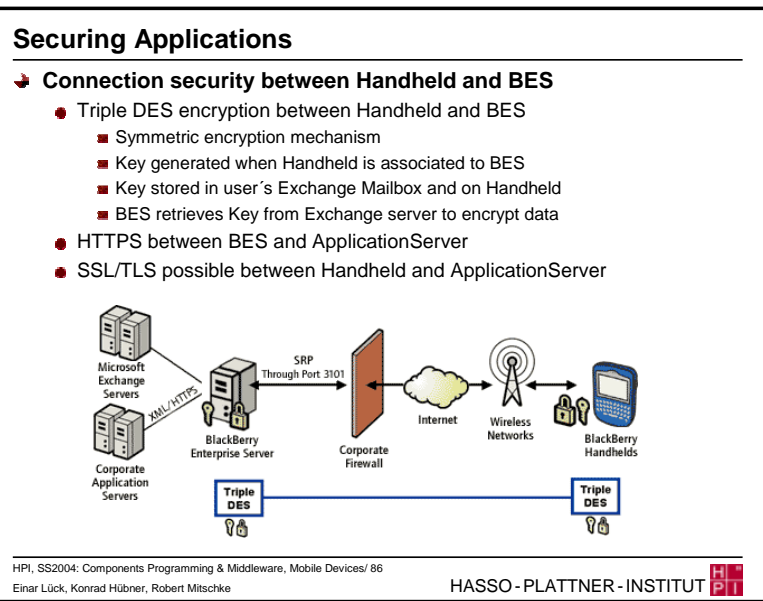

\footnotetext{
Securing Applications

- Communication

- Support for HTTP and HTTPs via MIDP API's HttpConnection class

- MDS can act as proxy for the Handheld

- Blackberry encryption used between corporate network and Handheld

- S/MIME support

- private-public key system

- S/MIME support package required to transfer keys to Handheld

\section{+ Controlled APIs for system access}

- Access to controlled APIs requires digital signment by RIM - Encryption API

- Persistent storage API

- System APIs

- Without those APIs no communication, device access or storage possible

\section{Securing Handheld \\ - Handheld supports Password Authentication \\ - Only SHA-1 Hash stored on Handheld => even memory access cannot \\ reveal password \\ - Too many attempts result in Handheld memory erasure \\ - Admin can change Handheld password remotely \\ - Admin can lock or delete information from lost Handhelds \\ - Policies for third-party applications \\ - Restrictions on type of network connection (WAP, BES, serial, USB) \\ - Restrictions on connection target (internal / external)

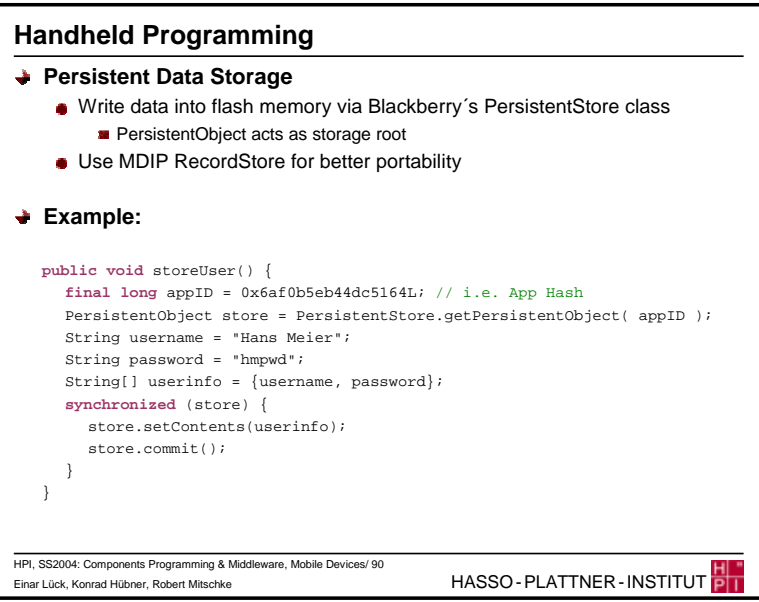



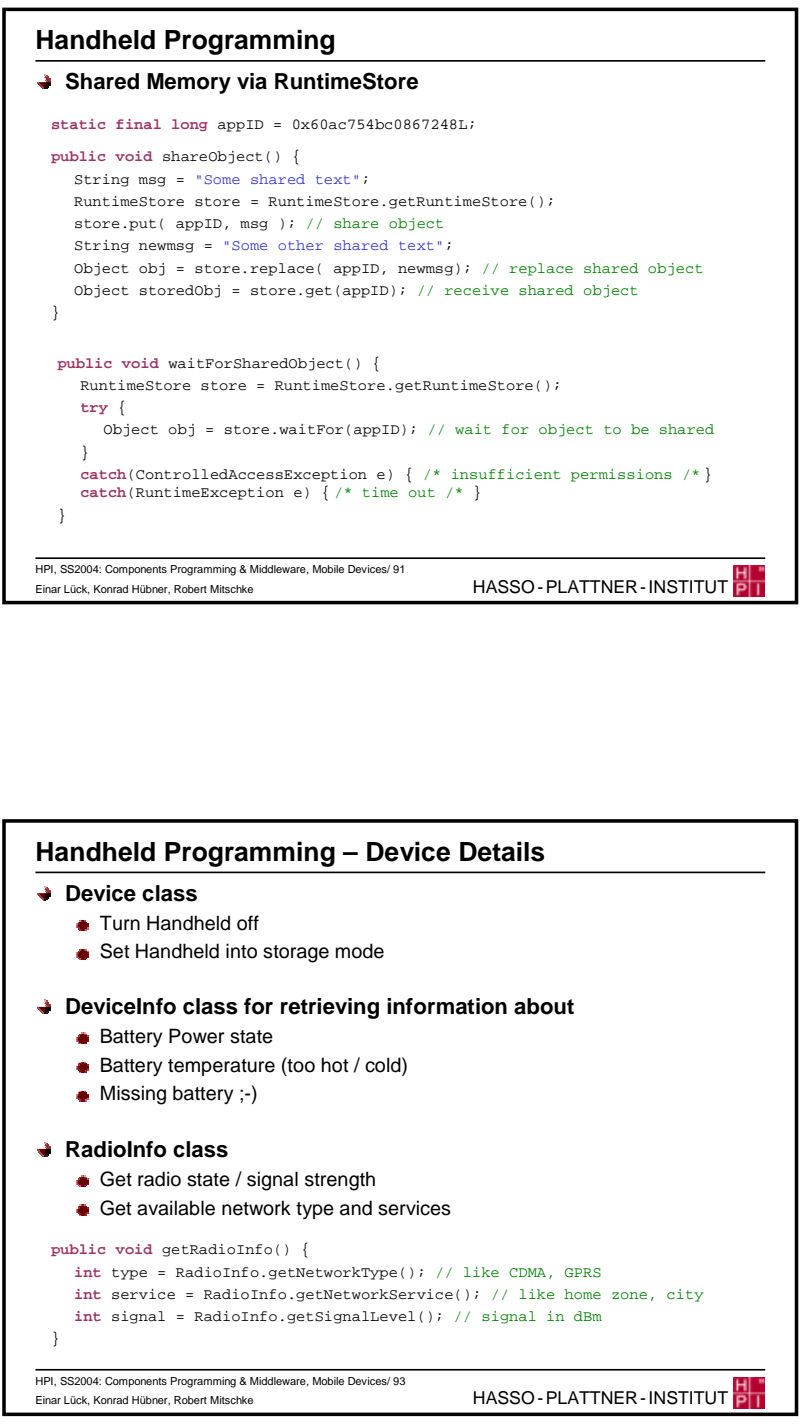

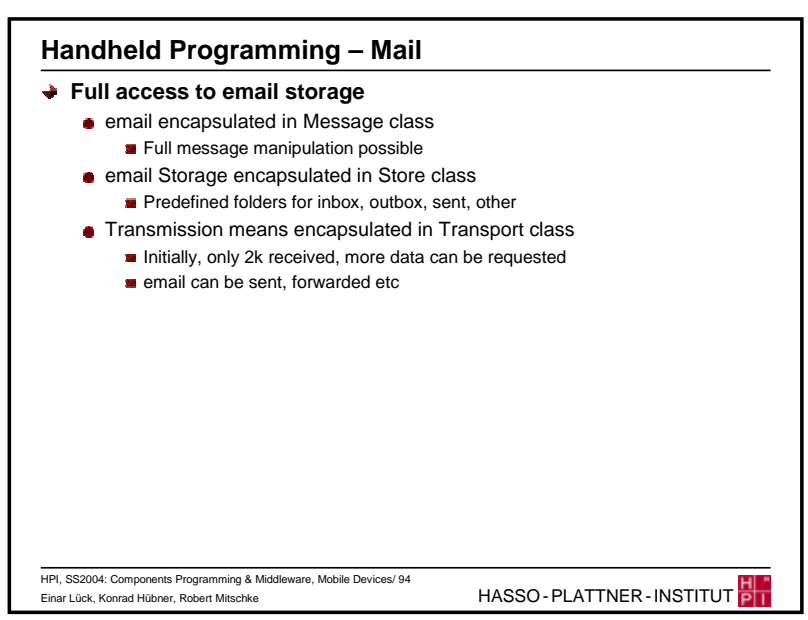

Handheld Programming - Low Memory Management

- In case of low memory old data is automatically removed

- Remote Address Lookup Requests

- Browser cache

- Old attachments

- Old calendar entries

- Old messages

- Calendar and emails are not sent back to the server but simply removed

\section{- Applications can}

- Register listeners for low-memory events

- Register objects for removal by VM in case of low memory

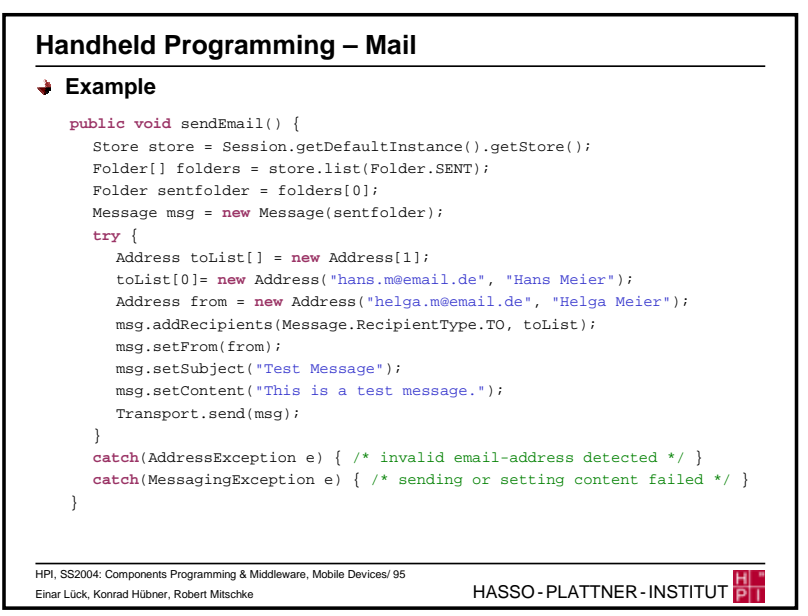

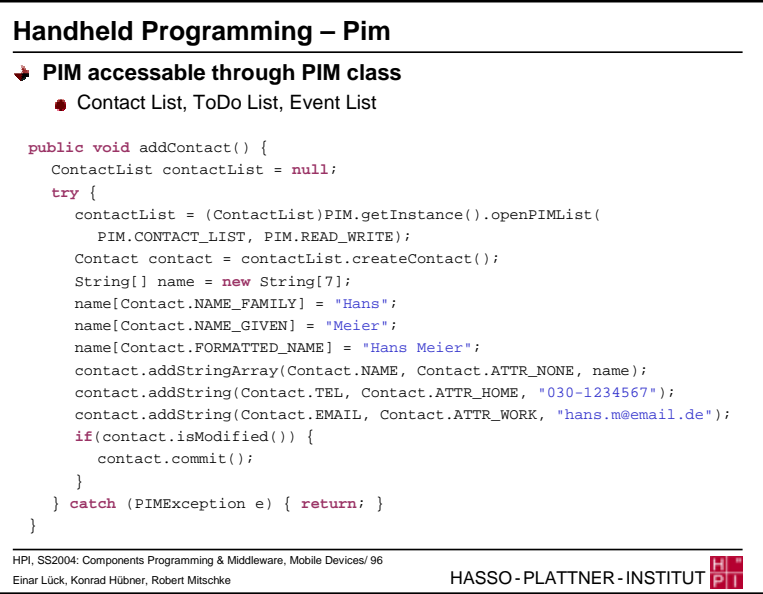




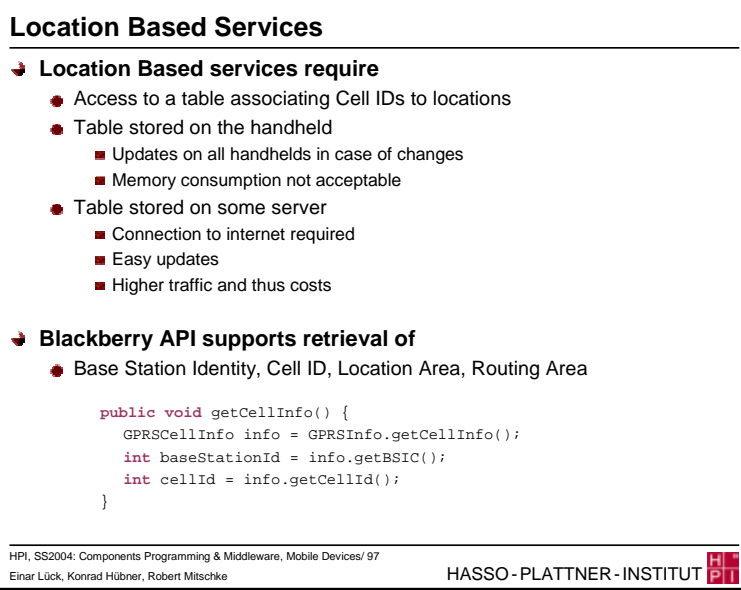

- Blackberry API supports retrieval of

- Base Station Identity, Cell ID, Location Area, Routing Area

public void getcellinfo() f

GPRSCellInfo info $=$ GPRSInfo.getcellinfo();

int basestationId $=$ info.getBSIC ()$;$

int cellId $=$ info.getcellId ()

HPl, SS2004: Components Programming \& Middleware, Mobile Devices/ 97
Enar Lock, Konrad Hubner, Robert Mischke
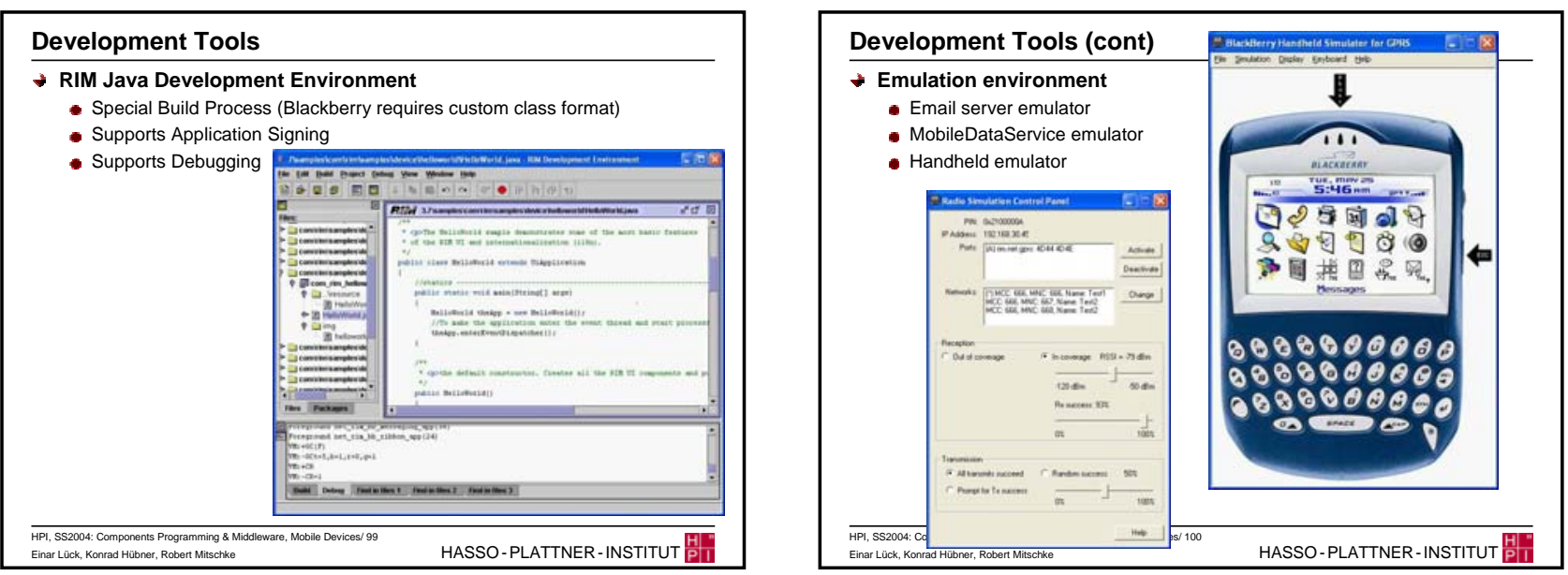

\section{Location Based Services II}

- Different Approach: use Carrier Services

- T-Mobile offers HTTPS interface to retrieve location

Latitude, Longitude

- Point

- City Area Code

\section{- Common Problems}

- Cell size ranges from $500 \mathrm{~m}$ in cities to $30 \mathrm{~km}$ on flat land

- Position within cell cannot easily be determined
- Circle (+ Radius)

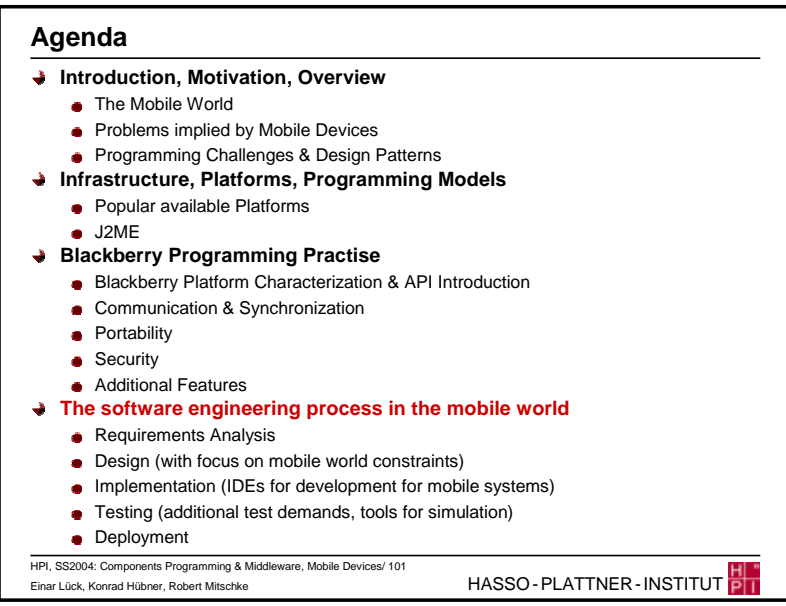

Requirements Analysis - Challenges

- Special mobile application requirements

- Dynamic environment

a User system interaction is fluid

- Contextual Applications

- Applications are highly related to the context of where they are in - Limited Attention

- Users can not pay attention at all times

- May be jogging, running, driving ..

- Short intervals of activity

- Applications are usually used often but only for short durations

- Users do not know what they want

- Some do not even know the target platform yet 


Requirements Analysis - Conduction
Conduction tips (see [14])
- Context based requirements gathering
- Get to know the environment / real life where application is used
- Prototyping!!!
- Key to success mobile phone while jogging / driving
- Educates users (they may learn to define what they want)
- Educates developers (they may learn what is possible and what is not)

Implementation - IDE showcase - EclipseME

\section{* Eclipse with EclipseME}

- Well known Java IDE extended by a plug-in

- Simplifies selection of APIs (Profile, Configuration, OEM)

- Allows Eclipse to be used for creating code

- Runs Simulators directly from Eclipse allowing easy testing and debugging

- Supports

- Java Wireless Toolkit including built in emulators

- Sony Ericsson J2ME SDK 20

- Nokia Developer Suite 2.0 for J2ME

- No support for Siemens platforms (can be used with plain MIDlets though)

- No support for Blackberry platform due to differing byte code format

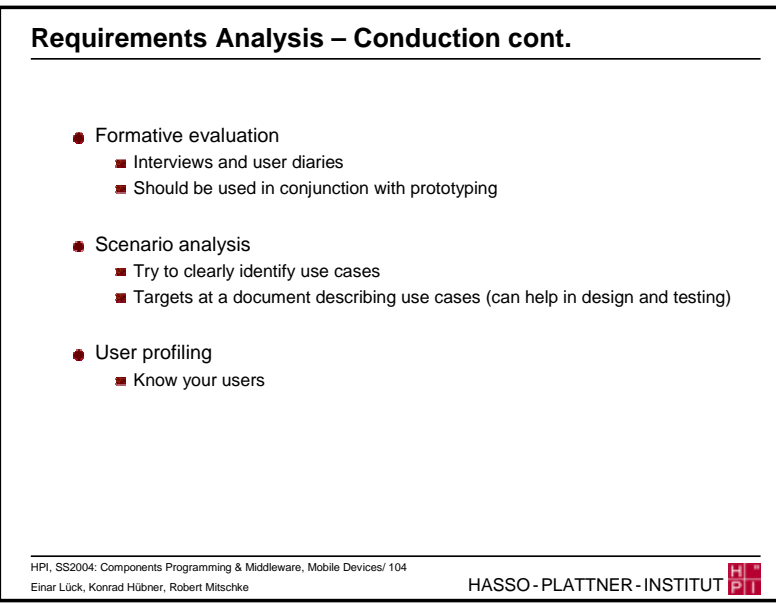

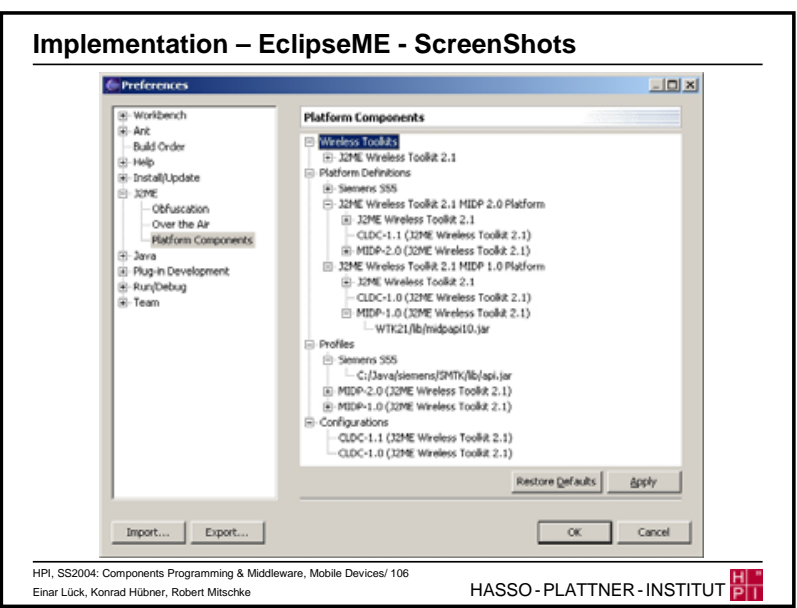

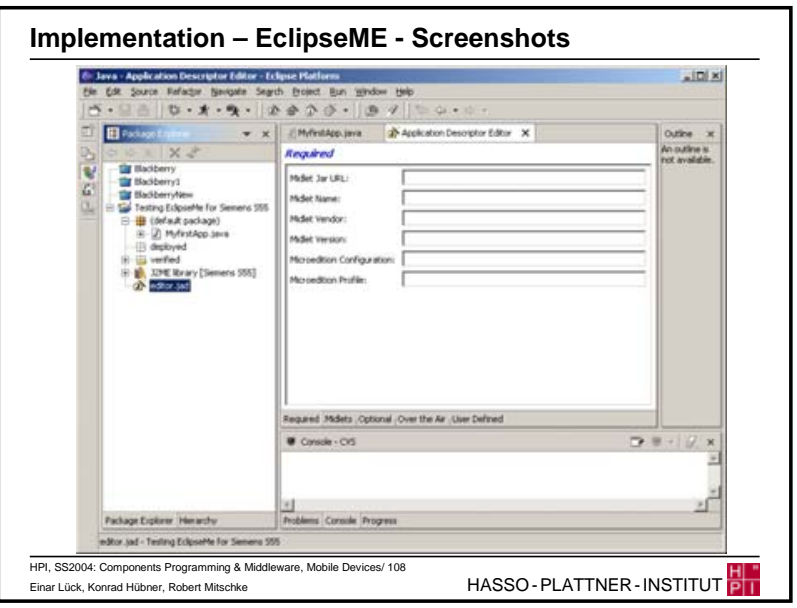


Implementation - IDE showcase - J2WT

+ Sun J2ME Wireless Toolkit 2.1

- Basic Toolkit used for plain MIDlet programming - No OEM APIs supported

- Includes device simulators for imaginary devices - Different screen sizes, colour depth and input devices - Supports detailed configuration of simulated environment
(e.g. connectivity, cpu speed, memory available ...)

Implementation - J2WT - Screenshots

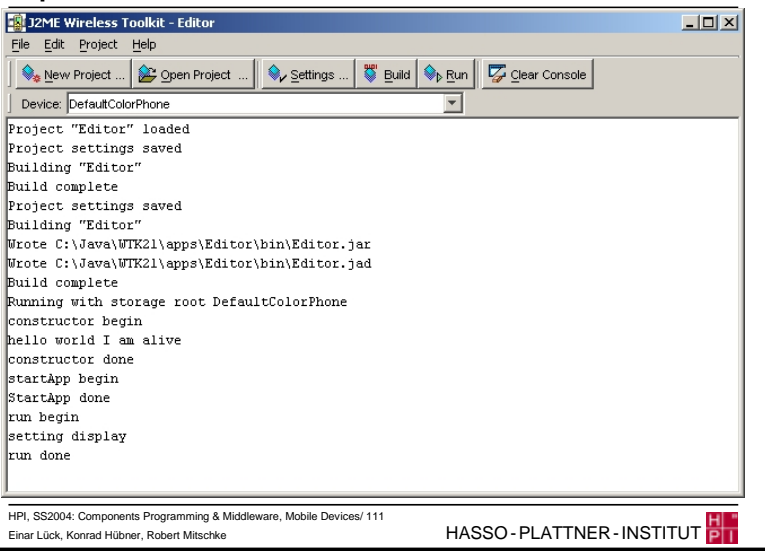

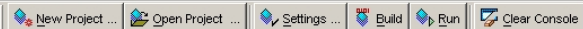

Device: Defautclolor Phone

Build comple

Jrote C: \JavalWTK2

constructor begin

constructor done

Startapp do

run begin

Einar Luck, Konrad Hüber, Robert Mischlye

Implementation - IDE showcase - Siemens

\section{- Siemens Mobility Toolkit}

- Consists of Device Simulators

- Support for debugging tracing

- Limited support for power constraints (supports power low mode)

- Support for SMS reception

- No support for network reliability configuration

- Support for IDE platforms limited to two professional non free IDEs - JBuilder Enterprise

Sun One Studio

- As they are non free they are not presented here
Implementation - IDE showcase - J2WT cont.

- Includes means for compilation, verification and packaging

* No real IDE, no code editing possible

- Instead rich set of additional tools

- Memory monitor

- Network Monitor

- Profiler

- Security (MIDP 2.0 trusted MIDlets) supported

- OTA provisioning sniffer

Implementation - J2WT - Screenshots cont.

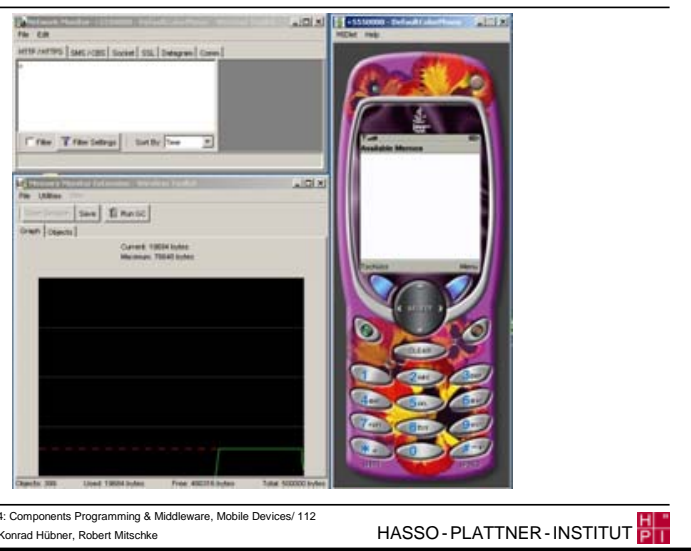

Implementation - IDE showcase - Blackberry IDE 


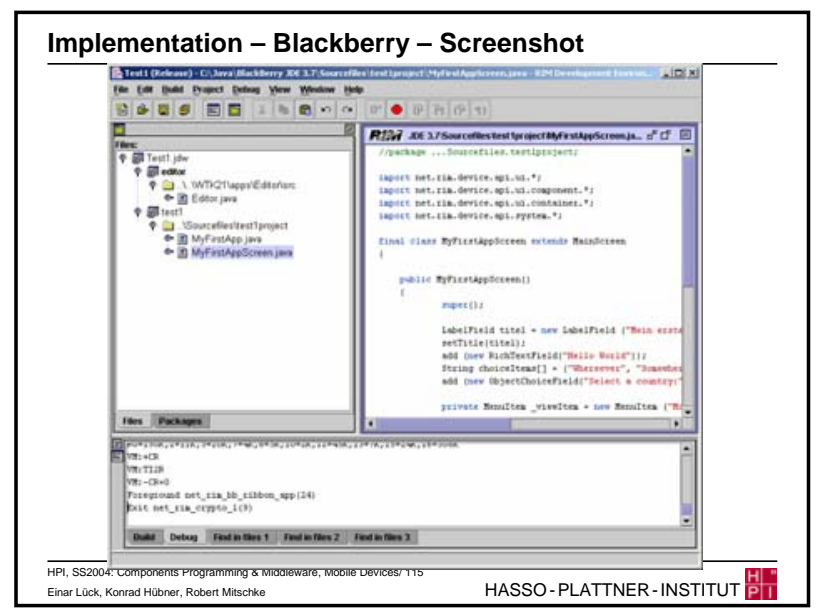

Testing - Demands

* Additional test demands

- Power consumption

- High utilization of CPU and other resources may lead to excessive battery drain

- User may not use application

- Battery may run low in wrong situation $\rightarrow$ user may be mad

\section{- Memory consumption}

- Need for memory of application needs to be evaluated before release

$\rightarrow$ allow use of application on various limited devices

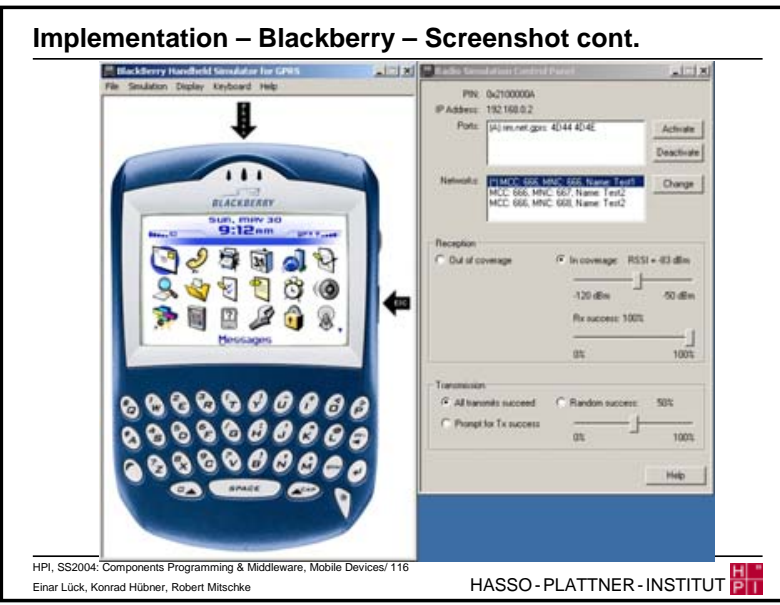

Testing - Demands cont.

- Behaviour with variable bandwidth and coverage / connectivity - Application needs to be tested to be robust against:

- Loss of single packet or packet bursts - Loss of connectivity (e.g. application should not lock for ever while waiting for
an ACK)

- Look and feel on different screen types

- Screen dimensions (Layout manager, own algorithm)

- Screen depth (colour, grey scale, black and white)

- Input devices

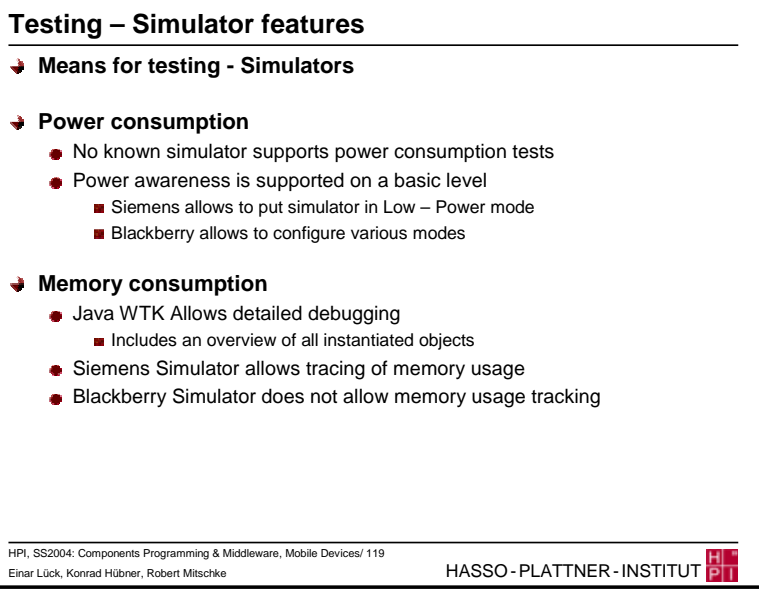

Testing - Simulator features cont.

* Bandwidth and coverage
- Very well supported in J2WT and Blackberry

a J2WT supports detailed overview of all network transfer, up to http level

- No support found in Siemens simulator

+ Look \& Feel and Input devices

- Best accomplished by testing on multiple platforms

- JWTK comes with different simulators for imaginary devices

- Siemens toolset allows for easy addition of multiple Siemens device

simulators (S55, SL55, MC60 ...)

+ Profiling

- Supported very well by J2WT

- Siemens covers profiling in trace files

- No support found in Blackberry

HPI, SS2004: Components Programming \& Middleware, Mobile Devicess 120 HASSO-PLATTNER-INSTITUT ${ }_{\text {P }}^{\text {H }}$ 


\section{Testing - In Device Debugging}

+ In device debugging

- Most Tool Kits try to minimize need for in device debugging Siemens and Blackberry Simulator use identical software in simulator and device by only porting a hardware abstraction to Windows platform - No in device debugging for JWTK (:)

No known support for Siemens phones

- In-device debugging support

- Potentially possible by using interfaces like RS232, USB, Bluetooth - Only if separated operating system support is present

Blackberry supports in-device debugging using jacket

- Cool parts of Blackberry API only work in device when signed

- You pay for key necessary to sign code

- Can be very tedious

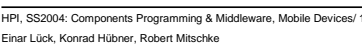
HASSO-PLATTNER-INSTITUT P PI

\section{Deployment}

\section{- OTA (Over The Air provisioning)}

- Defined in MIDP 2.0 - thus identical for all devices

- Blackberry supports application push via BES

- Own byte-code format requires conversion of J2ME apps

- Deployment directly onto device

- Siemens

I RS232 Cable comes with device

Bluetooth and IRDA also supported

- Windows drivers allows integration into Windows Explorer

Drag and Drop of JAR-Files (Folders need to be created by user)

- Blackberry via Desktop Software (USB)

\section{Deployment - Security}

* Security

- On Blackberry application installation has to be approved by user

- OTA provisioning is mainly secured by law

- Provider of application is responsible for his code

- Some devices allow deployment of applications via other ways (cable Infrared, Bluetooth)

- Potential security threa

- Even though standards are strict total security is not achievable

- Different implementations of standard bear risks (S55 SMS exploit)

\section{Deployment Example - Siemens}

\section{References}

*1] M. Satyanarayanan, .Fundamental Challenges in Mobile Computing“, Carnegie Mellon University, 1995.

- [2] Gamma et. Al, „Design Patterns“, Addison Wesley, 1994

- [3] R. Rosenbaum, C. Tominski, „Pixels vs. Vectors: Presentation of Large Images on Mobile Devices", University of Rostock.

* [4]

[5]

[6] B. D. Noble, M. Satyanarayanan, "A Research Status Report on Adaption for Mobile Data Access", Carnegie Melon University, 1995.

- [7] J.J. Kistler, M. Satyanarayanan, "Disconnected Operation in the Coda file System", ACM Transactions on Computer Systems 10, 1992.

- [8] M. Ebling, L. Mummert, D. Steere, "Overcomming the Network Bottleneck in Mobile Computing", In Proceedings of The IEEE Workshop on Mobile Computing Systems and Applications, 1999. 


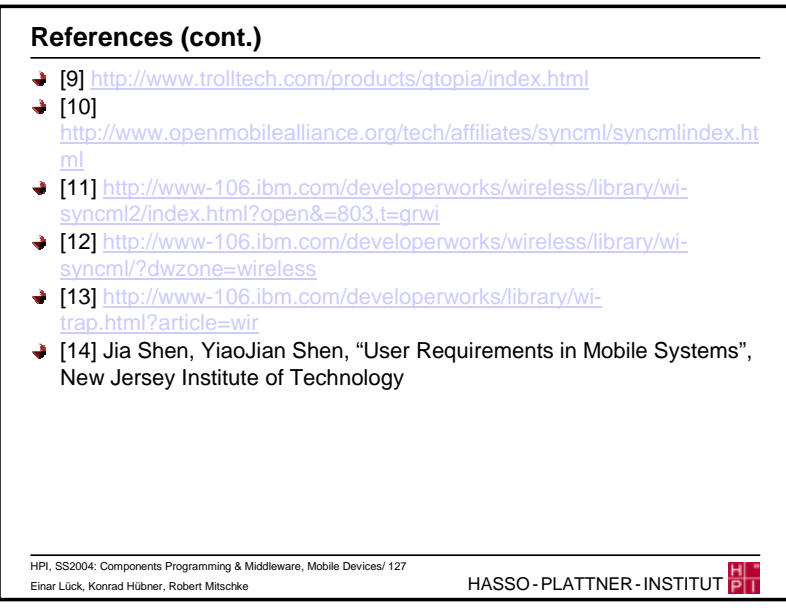

\section{References}

* [1] M. Satyanarayanan, „Fundamental Challenges in Mobile Computing “, Carnegie Mellon University, 1995.

[2] Gamma et. Al, „Design Patterns“, Addison Wesley, 1994.

- [2] Gamma et. Al, „Design Patterns“, Addison Wesley, 1994.
[3] R. Rosenbaum, C. Tominski, „Pixels vs. Vectors: Presentation of Large Images on Mobile Devices“, University of Rostock.

- [4]

$+[5]$

[6] B. D. Noble, M. Satyanarayanan, "A Research Status Report on Adaption for Mobile Data Access", Carnegie Melon University, 1995.

[7] J.J. Kistler, M. Satyanarayanan, "Disconnected Operation in the Coda file System", ACM Transactions on Computer Systems 10, 1992.

[8] M. Ebling, L. Mummert, D. Steere, “Overcomming the Network Bottleneck in Mobile Computing", In Proceedings of The IEEE Workshop on Mobile Computing Systems and Applications, 1999.

HPI, SS2004: Components Programming \& Middleware, Mobile Devices 1 12 HASSO-PLATTNER-INSTITUT ${ }^{\text {H }}$

References (cont.)

* [9] http://www.trolltech.com/products/qtopia/index.html

$+[10]$

[12] http://www-106.ibm.com/developerworks/wireless/library/wi-

[13] http: //wwww-106.ibm.com/developerworks/library/wi

+ [13] http://www-106.ibm.com/developerworks/library/u

- [14] http://sourceforge.net/projects/syncml-ctoolkit/ 